\title{
Performance of an ideal turbine in an inviscid shear flow
}

\author{
S. Draper ${ }^{1}$, T. Nishino ${ }^{2}$, T. A. A. Adcock ${ }^{3}$ and P. H. Taylor ${ }^{3}$ \\ ${ }^{1}$ School of Civil, Environmental and Mining Engineering, University of Western Australia, WA 6009, \\ Australia \\ ${ }^{2}$ Centre for Offshore Renewable Energy Engineering, Cranfield University, Bedfordshire MK43 0AL, UK \\ ${ }^{3}$ Department of Engineering Science, University of Oxford, Parks Road, OX1 3PJ, UK
}

\begin{abstract}
Although wind and tidal turbines operate in turbulent shear flow, most theoretical results concerning turbine performance, such as the well-known Betz limit, assume the upstream velocity profile is uniform. To improve on these existing results we extend the classical actuator disc model in this paper to investigate the performance of an ideal turbine in steady, inviscid shear flow. The model is developed on the assumption that there is negligible lateral interaction in the flow passing through the disc and that the actuator applies a uniform resistance across its area. With these assumptions, solution of the model leads to two key results. First, for laterally unbounded shear flow it is shown that the normalised power extracted is the same as that for an ideal turbine in uniform flow, if the average of the cube of the upstream velocity of the fluid passing through the turbine is used in the normalisation. Second, for a laterally bounded shear flow, it is shown that the same normalisation can be applied, but allowance must also be made for the fact that non-uniform flow bypassing the turbine alters the background pressure gradient and, in turn, the turbines 'effective blockage' (so that it may be greater or less than the geometric blockage, defined as the ratio of turbine swept area to cross-sectional area of the flow). Predictions based on the extended model agree well with numerical simulations approximating the incompressible Euler equations. The model may be used to improve interpretation of model-scale results for wind and tidal turbines in tunnels/flumes, to investigate the variation in force across a turbine and to update existing theoretical models of arrays of tidal turbines.
\end{abstract}

\section{Introduction}

The well-known actuator disc model was introduced by Lanchester, Betz and Joukowsky to estimate the performance of a wind turbine in laterally unbounded flow (van Kuik, 2007). The main feature of this model is that the complicated flow structures around the individual turbine blades are avoided by replacing the turbine with a permeable actuator disc that provides a uniform retarding force across its area. This simplification permits an analytical solution of the bulk flow velocity through the turbine and an estimate of the maximum power that can be extracted, referred to commonly as the Betz limit (Burton et al. 2001). However, despite these advantages, a well-known limitation of the classical actuator disc model is that it assumes that the flow is steady and spatially uniform upstream of the turbine. In practice this is rarely the case since turbines (both wind and tidal, for example) operate in turbulent flow that is sheared in the vertical and/or horizontal plane.

As a result of this limitation, detailed studies into the performance of turbines in turbulent shear flow have generally focussed on laboratory and field testing, as well as numerical modelling (see, for example, experimental work reviewed in Vermeer et al. 2003, and reviews on numerical modelling by Sørensen, 2011 and Sanderse et al. 2011). These more detailed approaches provide important insight into turbine performance in turbulent shear flow, including, for example, the description of blade force time histories (so as to estimate power generation and structural fatigue) and turbine wake characteristics (which may impact on downstream turbines in a farm and therefore farm efficiency). Nevertheless, the generation of experimental and numerical results is still much less efficient than using simple actuator 
disc models. Furthermore, most of these experimental and numerical results do not, in themselves, allow for straightforward interpretation of the relative contributions of shear and turbulence on the power performance of a turbine. Consequently, extrapolation of results obtained in one flow condition (with particular shear profile and turbulence characteristics) to a different flow condition is difficult. This is especially true for experimental and numerical results obtained in laterally bounded flows (as may be applicable for turbines in tunnels, flumes or shallow water flows) where it is known that geometric blockage effects will impact power performance (Garrett and Cummins, 2007; Houlsby et al. 2008; Whelan et al. 2009). However, blockage in sheared flow, and therefore the ability to extrapolate model results to full-scale conditions, is not well understood.

In light of these limitations, the primary aim of this paper is to improve interpretation and extrapolation of experimental and numerical modelling results for turbines in turbulent shear flow by investigating and quantifying the effect of shear, alone, on the performance of an ideal turbine. To work towards this aim we extend the classical actuator disc model to incorporate a steady, inviscid shear flow. This extension builds on earlier work presented in Draper et al. (2014) and considers both the problem of laterally unbounded flow, which may be most applicable to full-scale wind turbines, as well as laterally bounded flow, which allows for an investigation of blockage in sheared flow.

A second motivation for the work in this paper is to build on recent observations concerning the performance of turbines in shear flow. In particular, Wagner et al. (2011) and Fleming et al. (2013) have suggested that to properly compare the performance of turbines in different shear flows the traditional definition of the power coefficient should be altered. Specifically, they suggest that the average of the cube of the velocity passing through an upstream area equal to the turbine swept area should be used to calculate the coefficient, as opposed to the cube of another reference velocity (such as the velocity at the hub height of the turbine). Both Wagner et al. (2011) and Fleming et al. (2013) provide support for this suggestion by demonstrating that the corrected power coefficient appears to explain variations in power performance observed in field measurements of wind turbines and blade-resolved numerical simulations of tidal turbines, respectively. However, although these comparisons are promising, they are for turbines having small geometric blockage of $\sim 10 \%$ or less (where geometric blockage is defined as the ratio of turbine swept area to cross-sectional area of the flow) and so it is not yet clear if the correction is also appropriate for larger blockage ratios, as may be experienced in experimental arrangements or in tidal turbine arrays where large blockage ratios are known to be potentially beneficial (see, for example, Vennell, 2010). Furthermore, for laterally unbounded flow the suggested correction of Wagner et al. (2011) and Fleming et al. (2013) is not the same as a recent proposed correction derived theoretically by Chamorro and Arndt (2013). Therefore it appears that further investigation is required to understand turbine performance in both laterally unbounded and laterally bounded shear flow.

To incorporate shear flow into the classical actuator disc model we introduce two new assumptions in this paper. Firstly, since the upstream velocity profile is generally nonuniform it is no longer appropriate to assume (as is typical in the classical model) that the disc applies a uniform streamwise force on the fluid. We will therefore assume instead that a turbine can be represented by an ideal disc with uniform local resistance (i.e. uniform local drag coefficient, as opposed to a uniform force). This alternative definition is convenient because, although the axial resistance of actual turbines may vary over their radius, uniform resistance is a useful reference case and will be a reasonable first approximation for some turbines. It is also likely to be representative of porous discs, which are often used to emulate arrays of turbines in experiments (e.g. Myers and Bahaj, 2012) and is of relevance to the 
estimation of drag on offshore structures generally (following the seminal work of Taylor, 1991, for example).

Secondly, to model the flow passing through the actuator disc analytically we will assume (as is common in blade element momentum theory; Burton et al. 2001) that there is negligible lateral (or spanwise) interaction across the flow passing through the disc; i.e. we will assume that, as outlined in more detail in $\S 2$, the momentum balance in each annuli of fluid passing through the disc (or slab of fluid for a rectangular actuator strip) is not affected by streamwise flow expansion in neighbouring annuli. To validate this assumption we will compare the theoretical model with numerical simulations approximating the Euler equations in $\S 5$.

Making use of these two assumptions we extend the classical model in stages in the remainder of the paper, beginning with laterally unbounded flow and then considering the more general problem of laterally bounded flow. Following this we present solutions for some example shear flows, before comparing with numerical simulations. Throughout the paper we choose to focus on two-dimensional flows, in which the disc becomes a strip with a spanwise dimension $l$. This configuration is mathematically convenient and has application to practically important configurations of turbines (discussed further in §6).

\section{Laterally unbounded shear flow}

To incorporate shear flow in the classical actuator disc model we start by considering an actuator strip operating in a symmetric shear flow with streamwise velocity $u\left(x_{1}, z\right)=$ $u\left(x_{1},|z|\right)=u_{1}(|z|) \geq 0$ and no lateral boundary; the coordinates being defined so that $x_{1}$ is a location far upstream of the strip where the flow is undisturbed by the strip's presence and $z$ is a spanwise coordinate which takes a value of zero at the strip centre (figure 1). As in the classical actuator disc analysis we also assume that the flow is steady, incompressible and inviscid.

To extract power the strip must offer a resistance to the flow. Focusing on an infinitesimal control volume bounded by two neighbouring streamlines (or stream surfaces) and intersecting an area of strip $\delta l$ (per unit width), we can introduce this resistance as a force $\delta T$ on the fluid (figure 1). Because of this force, the fluid passing through the strip is reduced to $u\left(x_{2}, \psi\right)=u_{2}(\psi)=\alpha_{2}(\psi) u_{1}(\psi)$, where $\alpha_{2}(\psi) \in[0,1], u_{1}(\psi)$ is the upstream velocity entering the control volume and $\psi$ is the average value of the stream function defining the stream surfaces bounding the control volume (see figure 1b). Far downstream of the strip, where the static pressure is constant for any $\psi$, the velocity in the control volume reduces further to $u\left(x_{4}, \psi\right)=u_{4}(\psi)=\alpha_{4}(\psi) u_{1}(\psi)$, with $\alpha_{4}(\psi) \in\left[0, \alpha_{2}(\psi)\right]$.

With this problem definition we can now relate the velocity coefficients within the control volume to the force applied by the strip and, in turn, the power removed by the strip. This analysis proceeds in the same way as in the classical actuator disc theory, except that we will focus here on the differential fluid element within the control volume, rather than the whole disc/strip. To do this we start by using an argument of mass conservation to deduce that

$$
\delta l_{1}=\alpha_{2}(\psi) \delta l \quad \text { and } \quad \delta l_{4}=\left(\alpha_{2}(\psi) / \alpha_{4}(\psi)\right) \delta l,
$$

where $\delta l_{1}$ and $\delta l_{4}$ define the lateral width of the control volume far upstream and far downstream of the strip, respectively. Next, we can apply the Bernoulli equation separately upstream and downstream of the strip to obtain an expression for the pressure difference across the strip

$$
\Delta p(\psi)=p\left(x_{2}, \psi\right)-p\left(x_{3}, \psi\right)=\frac{1}{2} \rho\left[u_{1}(\psi)\right]^{2} \times\left(1-\left[\alpha_{4}(\psi)\right]^{2}\right),
$$


where $x_{2}$ and $x_{3}$ are locations immediately upstream and downstream of the strip, $p$ represents

(a)

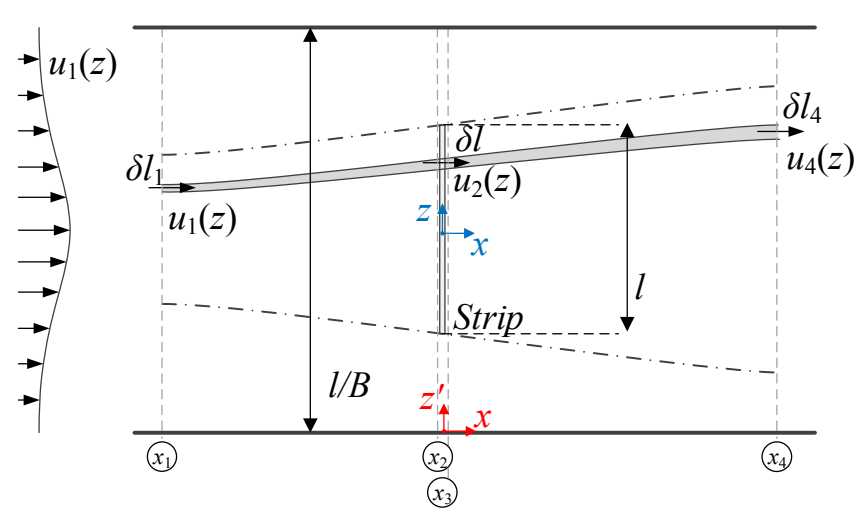

(b)

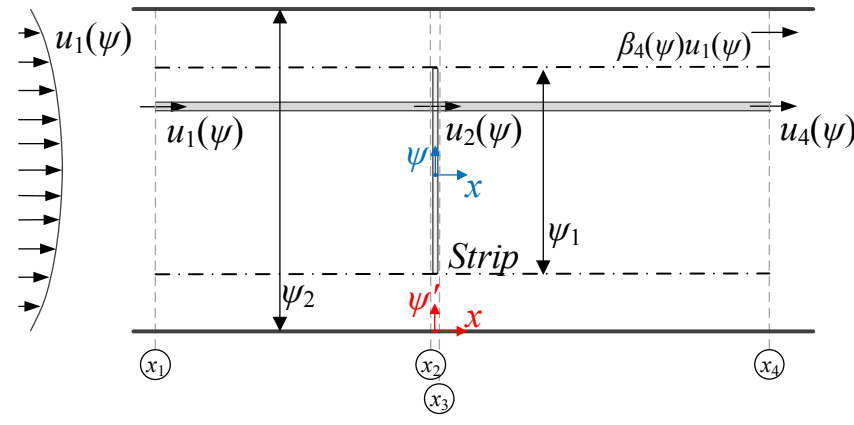

Figure 1: Assumed flow field through a strip. (a) $x-z$ plane; (b) $x-\psi$ plane. The solid horizontal lines indicate lateral boundaries, and their location is defined in terms of the geometric blockage ratio $B$ (introduced in $\S 3$ ). For laterally unbounded flow $B=0$. The translated $x-z^{\prime}$ and $x-\psi^{\prime}$ coordinate systems are used in $\S 4.2$.

static pressure and $\rho$ is fluid density. Finally, to complete the analysis, conservation of streamwise momentum (for the control volume) leads to

$$
-\delta T=\rho u_{1}(\psi) \alpha_{2}(\psi) \delta l\left[\left(\alpha_{4}(\psi)-1\right)\right] u_{1}(\psi)-\delta X,
$$

where

$$
\delta X=\oint_{C V} p_{x} d s
$$

and $p_{x}$ is the streamwise component of pressure acting normal to the surface of the control volume.

Noting that $\Delta p=\delta T / \delta l$ (to satisfy static equilibrium across the strip) equations (2.1), (2.2) and (2.3) can be combined to obtain a relationship between the velocity coefficient at the strip and the coefficient in the wake. This relationship is equivalent to that given by Sørensen (2011) (neglecting azimuthal velocity) and can be written as

$$
\alpha_{2}(\psi)=\frac{1+\alpha_{4}(\psi)}{2}\left(1-\frac{\delta X}{\delta T}\right)
$$

Importantly, because we have focused on a differential fluid element, the result in (2.5) is applicable at any location on the strip and is therefore a more general result than that which may be obtained by enclosing the entire actuator strip within a control volume. Interpreted 
directly, equation (2.5) indicates that at any given location on the strip the velocity is equal to the average of the upstream and downstream velocity on the same streamline only if there is no net forcing due to the pressure acting on the surface of the control volume. Goorjian (1972) first outlined that this net forcing will not be zero in general, whilst more recently Sørensen (2011) has also reported that $\delta X / \delta T$ can reach $5 \%$ for a disc in uniform flow. Nevertheless, as mentioned in the Introduction, it is common in blade element momentum theory to assume that $\delta X=0$ in (2.5) for all fluid elements passing through the disc (Sørensen, 2011). This is equivalent to assuming that the fluid within each control volume is able to expand independently of neighbouring control volumes as it passes through the strip. Partly due to simplicity, and partly due to the general success of blade element momentum theory in practice, we adopt this same assumption of lateral (or spanwise) independence in this paper. As noted in the Introduction, comparisons with numerical simulations are presented in $\S 5$ to validate this assumption in the context of shear flow.

Taking $\delta X=0$ in (2.5) it is now straightforward to obtain an expression for the power removed by the entire strip (per unit width), which is

$$
\begin{gathered}
P=\int_{-l / 2}^{l / 2} u_{2}(z) \Delta p(z) d z=\int_{-\psi_{1} / 2}^{\psi_{1} / 2} \Delta p(\psi) d \psi \\
=\frac{1}{2} \rho \int_{-\psi_{1} / 2}^{\psi_{1} / 2} 4\left[u_{1}(\psi)\right]^{2}\left(\alpha_{2}(\psi)-\left[\alpha_{2}(\psi)\right]^{2}\right) d \psi,
\end{gathered}
$$

where continuity has been used to write the result in terms of the stream function (i.e. we have converted to stream function coordinates by noting that $\partial \psi / \partial z=u$ ) and the parameter $\psi_{1}$ represents the total volume flux passing through the strip, which is given implicitly by

$$
l=\int_{-l / 2}^{l / 2} d z=\int_{-\psi_{1} / 2}^{\psi_{1} / 2} \frac{d \psi}{\alpha_{2}(\psi) u_{1}(\psi)}
$$

Presuming that $\alpha_{2}(\psi)$ is known at all points on the strip, (2.6) and (2.7) provide the general solution for the extracted power (assuming lateral independence) in a steady, inviscid shear flow. Alternatively, if a local disc resistance $k(\psi)$ is defined such that

$$
\Delta p(\psi)=\frac{1}{2} \rho k(\psi)\left[u_{2}(\psi)\right]^{2},
$$

(i.e. for a space-frame structure $k$ is equivalent to the product of a local Morison-type quadratic drag coefficient and the ratio of hydrodynamic to frontal area of the obstacle; Taylor 1991; Santo et al. 2014) the results in (2.6) and (2.7) can be rewritten in terms of the local resistance as

$$
P=\frac{1}{2} \rho \int_{-\psi_{1} / 2}^{\psi_{1} / 2} \frac{16 k(\psi)}{(k(\psi)+4)^{2}}\left[u_{1}(\psi)\right]^{2} d \psi, \text { with } \quad l=\frac{1}{4} \int_{-\psi_{1} / 2}^{\psi_{1} / 2} \frac{k(\psi)+4}{u_{1}(\psi)} d \psi .
$$

In the remainder of this section we investigate the power removed by the strip by solving equation (2.9). To start, we consider our main scenario in which $k$ is assumed to be constant across the strip; i.e. the strip has uniform resistance. For this case it follows immediately from (2.5) (with $\delta X=0$ ), and from comparing (2.2) with (2.8), that $\alpha_{2}$ and $\alpha_{4}$ are also constant in the spanwise direction; i.e. the velocity profile is self-similar at locations upstream, 
downstream and at the turbine when the local resistance is uniform. It is therefore possible to convert (2.9) into an integral upstream of the strip, such that

$$
P=\frac{1}{2} \rho \frac{16 k}{(k+4)^{2}} \int_{-\psi_{1} / 2}^{\psi_{1} / 2}\left[u_{1}(\psi)\right]^{2} d \psi=\frac{1}{2} \rho \frac{16 k}{(k+4)^{2}} \int_{-l_{1} / 2}^{l_{1} / 2}\left[u_{1}(z)\right]^{3} d z .
$$

Rearranging (2.10), noting that $l_{1}=\alpha_{2} l=4 l /(k+4)$, gives the power coefficient:

$$
C_{P}=\frac{P}{\frac{1}{2} \rho l \overline{U_{*}^{3}}}=\frac{64 k}{(k+4)^{3}}, \text { with } \overline{U_{*}^{3}}=\frac{1}{l_{1}} \int_{-l_{1} / 2}^{l_{1} / 2}\left[u_{1}(z)\right]^{3} d z .
$$

This power coefficient varies with disc resistance in exactly the same way as the power coefficient for a disc in uniform flow and is a maximum when $k=2$ so that $C_{P, \max }=16 / 27$. Equation (2.11a) therefore demonstrates that for a uniform disc in an inviscid shear flow the power coefficient is identical to that in uniform flow, provided that the average of the cube of the upstream velocity of the fluid passing through the disc is used in the normalisation (i.e. $\left.\overline{U_{*}^{3}}\right)$. This result is different to that introduced by Wagner et al. (2011) and Fleming et al. (2013), who suggested the integral in $(2.11 b)$ should be in terms of the upstream velocity evaluated over the full area of the strip (i.e. $l$ instead of $l_{1}$ ). We note, though, that in most practical situations, performing the upstream integration over the full strip area $l$ will give similar results to $(2.11 a)$, especially for actuators with small local resistance or for shear flow that is close to uniform across the plane of the strip. The result in (2.11) is also different to that given by Chamorro and Arndt (2013). This difference arises because here we focus on an actuator strip with uniform local resistance, whereas in Chamorro and Arndt (2013) their analysis requires that the pressure difference $\Delta p$, and therefore the force per unit frontal area, is uniform across the actuator (see, for example, their equation 9 and 15). Consequently their result is intended for turbines or porous obstructions that provide a uniform force in nonuniform flow (which would be possible for a particular variation of flow resistance across the actuator).

Returning to equation (2.9), it can be seen that for more complicated scenarios in which the local disc resistance is allowed to vary, the optimum distribution of local resistance is more difficult to determine in non-uniform flow. This is because although the momentum balance within each control volume is assumed to be independent, power can only be extracted across the finite area of the strip. This interdependence is captured in $(2.9 a)$ in terms of the upper limit to the integration $\psi_{1}$; i.e. $k(\psi)$ should be chosen not only to maximise the integrand in the equation for power, but also to ensure a significant collective flow rate through the disc $\psi_{1}$. We do not attempt to solve (2.9) in this paper for a non-uniform resistance. However we do remark that the optimum resistance will be non-uniform in a non-uniform flow. We also remark that the assumption of lateral independence may become less plausible in some scenarios if the resistance is being altered to significantly manipulate the flow through the strip (rather than allowing free expansion).

Before concluding this section we note that up until now our analysis has been restricted to a two-dimensional problem in symmetric shear flow. However, provided $\overline{U_{*}^{3}}$ is calculated by averaging over the area of flow passing through the actuator, equation (2.11) is valid for asymmetric and three-dimensional flow. This is because (2.11) is simply an integral across independent fluid elements passing through the strip/disc, and these elements may be defined regardless of flow symmetry or strip/disc geometry. The only complication in practice is that the application of (2.11) requires knowledge of the upstream location of the flow passing through the strip/disc. In general this location may not be concentric to the strip/disc in 
asymmetric flow, and so the upstream location is difficult to define without mapping the outermost streamlines based on a complete solution for the upstream velocity field. We return to this difficulty in $\S 4.2$ and $\S 5$.

\section{Laterally bounded shear flow}

We now extend the analysis to consider the more general problem of an actuator strip in laterally bounded flow. To do this we adopt the same assumptions as in $\S 2$, except that we confine the flow between two parallel walls separated by a distance $l / B$; where $B$ defines the geometric blockage ratio (figure 1). In addition to the velocity coefficients $\alpha_{2}(\psi)$ and $\alpha_{4}(\psi)$ we also introduce a new velocity coefficient $\beta_{4}(\psi) \geq 1$ to define the streamwise velocity of the fluid bypassing the strip. Finally, as in earlier actuator disc models, we will choose to neglect frictional forces acting on the lateral walls. In practice these forces would, of course, be responsible for establishing the shear in the flow. However the assumption inherent in our analysis is that inertia forces dominate these frictional forces over the length scale of streamwise flow diversion around the strip (i.e. over the distance $L=x_{4}-x_{1}$ ).

With this problem definition it is again possible to establish a relationship between the local velocity coefficients and, in turn, the power removed by the strip. However, to make the analysis simpler in the following sections we will restrict our analysis from here on to a scenario in which $\alpha_{2}$ and $\alpha_{4}$ are uniform across the strip and the wake respectively; i.e. we will assume the velocity profile passing through the strip is self-similar upstream, downstream and at the strip. As outlined in $\S 2$ for unbounded flow, if we assume spanwise independence then setting both of these coefficients to be uniform implies a strip having a uniform local resistance. Similarly, for laterally bounded flow (i.e. $B>0$ ) we will see later in this Section that although uniform $\alpha_{2}$ and $\alpha_{4}$ does not strictly imply that the local strip resistance is uniform, in most realistic scenarios it will be very close to uniform. This means that, as intended, the solutions to be derived below are appropriate for estimating the performance of an ideal strip with uniform resistance.

Having made this simplifying assumption we can now begin the analysis by determining the geometry of the streamlines that enclose the flow passing through the strip. From continuity, the lateral distance between these streamlines upstream and downstream of the strip are

$$
l_{1}=\int_{-\psi_{1} / 2}^{\psi_{1} / 2} \frac{d \psi}{u_{1}(\psi)}, \quad \text { and } \quad l_{4}=\frac{1}{\alpha_{4}} \int_{-\psi_{1} / 2}^{\psi_{1} / 2} \frac{d \psi}{u_{1}(\psi)}
$$

At the disc we can also write

$$
l=\int_{-l_{l} / 2}^{l / 2} d z=\int_{-\psi_{1} / 2}^{\psi_{1} / 2} \frac{d \psi}{u_{2}(\psi)}=\frac{1}{\alpha_{2}} \int_{-\psi_{1} / 2}^{\psi_{1} / 2} \frac{d \psi}{u_{1}(\psi)} .
$$

where, again, $\psi_{1}$ is the volume flux passing through the strip.

With these results we can now integrate over the cross-sectional area of the flow bypassing the strip to arrive at an expression to relate all of the velocity coefficients

$$
\frac{l}{B}-\frac{\alpha_{2}}{\alpha_{4}} l=\int_{-\psi_{2} / 2}^{-\psi_{1} / 2} \frac{d \psi}{\beta_{4}(\psi) u_{1}(\psi)}+\int_{\psi_{1} / 2}^{\psi_{2} / 2} \frac{d \psi}{\beta_{4}(\psi) u_{1}(\psi)},
$$

where $\psi_{2}$ is the volume flux for the entire confined flow; i.e. 


$$
\psi_{2}=\int_{-l / 2 B}^{l / 2 B} u_{1}(z) d z
$$

To continue the analysis we now apply the Bernoulli equation separately upstream and downstream of the strip, which leads to the following expression for the pressure change across the strip

$$
\Delta p(\psi)=p\left(x_{2}, \psi\right)-p\left(x_{3}, \psi\right)=\Delta p^{\prime}+\frac{1}{2} \rho\left[u_{1}(\psi)\right]^{2} \times\left(1-\alpha_{4}^{2}\right) .
$$

This expression is the same as (2.2) for the unbounded case, except that it is augmented by the background pressure difference $\Delta p^{\prime}$. This background pressure difference should be uniform laterally across the flow and may be obtained by writing the Bernoulli equation along any streamline in the bypass flow, which leads to

$$
\Delta p^{\prime}=\frac{1}{2} \rho\left[u_{1}(\psi)\right]^{2}\left(\left[\beta_{4}(\psi)\right]^{2}-1\right), \quad \text { for } \quad \psi_{2} \geq|2 \psi| \geq \psi_{1} .
$$

Finally, to complete the analysis we can enforce conservation of streamwise momentum across the entire flow field, which leads to

$$
\Delta p^{\prime} \frac{l}{B}-T=\rho \int_{-l / 2 B}^{l / 2 B}\left[u_{4}(z)\right]^{2} d z-\rho \int_{-l / 2 B}^{l / 2 B}\left[u_{1}(z)\right]^{2} d z
$$

where the force supplied by the strip is

$$
T=\int_{-l / 2}^{l / 2} \Delta p(z) d z
$$

Substituting (3.5) and (3.8) into (3.7), and separating the integrals on the right-hand side of (3.7), the momentum equation can be rewritten as

$$
\begin{aligned}
\Delta p^{\prime} \frac{l}{B}-\Delta p^{\prime} l & -\frac{\rho\left(1-\alpha_{4}^{2}\right)}{2 \alpha_{2}} \int_{-\psi_{1} / 2}^{\psi_{1} / 2} u_{1}(\psi) d \psi=\rho\left(\alpha_{4}-1\right) \int_{-\psi_{1} / 2}^{\psi_{1} / 2} u_{1}(\psi) d \psi \\
& +\rho \int_{-\psi_{2} / 2}^{-\psi_{1} / 2}\left(\beta_{4}(\psi)-1\right) u_{1}(\psi) d \psi+\rho \int_{\psi_{1} / 2}^{\psi_{2} / 2}\left(\beta_{4}(\psi)-1\right) u_{1}(\psi) d \psi .
\end{aligned}
$$

If we now presume that the velocity coefficient $\alpha_{2}$ is known, together with the geometric blockage $B$ and the upstream velocity profile $u_{1}(\psi)$, equations (3.3) and (3.9) define a set of two equations which may be solved together to determine the unknown coefficient $\alpha_{4}$ and the unknown function $\beta_{4}(\psi)$. To obtain this solution it is convenient to first reduce the function $\beta_{4}(\psi)$ to a single parameter. This is possible due to the fact that the background pressure difference must be the same along all streamlines in the bypass flow. Hence, from (3.6)

$$
\beta_{4}(\psi)=\left[1+\frac{u_{1}^{\prime 2}}{\left[u_{1}(\psi)\right]^{2}}\left(\beta_{4}^{\prime 2}-1\right)\right]^{1 / 2}, \quad \text { for } \quad \psi_{2} \geq|2 \psi| \geq \psi_{1},
$$


where $\beta_{4}^{\prime}=\beta_{4}\left(\psi_{b}\right)$ and $u_{1}^{\prime}=u_{1}\left(\psi_{b}\right)$ have been introduced to represent the bypass velocity coefficient and upstream velocity, respectively, along an arbitrary streamline $\psi=\psi_{b}$ in the bypass flow. Since $\psi_{b}$ can be prescribed, it follows that $u_{1}^{\prime}$ is a known parameter and the function $\beta_{4}(\psi)$ is defined, via (3.10), in terms of only the single unknown parameter $\beta_{4}^{\prime}$.

Rewriting (3.3) and (3.9), using (3.10), now leads to the following two equations

$$
\alpha_{4}=\frac{\alpha_{2} \beta_{4}^{\prime} B}{\alpha_{2} B I_{0}+\beta_{4}^{\prime}-I_{0}} .
$$

and

$$
\begin{aligned}
& (1-B) \beta_{4}^{\prime 2}-2\left(I_{2}-\alpha_{4}\left[\frac{I_{1}\left(\alpha_{4}-1\right)-I_{2}\left(\beta_{4}^{\prime}-1\right)}{I_{0} \alpha_{4}-\beta_{4}^{\prime}}\right]\right) \beta_{4}^{\prime}+ \\
& \left(1+2\left(I_{2}-1\right)+B\left(1-I_{1}\right)-2 \alpha_{4}\left[\frac{I_{1}\left(\alpha_{4}-1\right)-I_{2}\left(\beta_{4}^{\prime}-1\right)}{\alpha_{4}-\beta_{4}^{\prime} / I_{0}}\right]+B \alpha_{4}^{2} I_{1}\right)=0,
\end{aligned}
$$

in which $I_{0}, I_{1}$ and $I_{2}$ are given by

$$
\begin{gathered}
I_{0}=\frac{B \beta_{4}^{\prime}}{\left(1-B \alpha_{2}\right) l} \int_{-\psi_{2} / 2}^{-\psi_{1} / 2}\left(\left[u_{1}(\psi)\right]^{2}+u_{1}^{\prime 2}\left(\beta_{4}^{\prime 2}-1\right)\right)^{-\frac{1}{2}} d \psi \\
+\frac{B \beta_{4}^{\prime}}{\left(1-B \alpha_{2}\right) l} \int_{\psi_{1} / 2}^{\psi_{2} / 2}\left(\left[u_{1}(\psi)\right]^{2}+u_{1}^{\prime 2}\left(\beta_{4}^{\prime 2}-1\right)\right)^{-\frac{1}{2}} d \psi, \\
I_{1}=\frac{1}{u_{1}^{\prime 2} \alpha_{2} l} \int_{-\psi_{1} / 2}^{\psi_{1} / 2} u_{1}(\psi) d \psi,
\end{gathered}
$$

and

$$
\begin{gathered}
I_{2}=\frac{B}{\left(1-B \alpha_{2}\right) l u_{1}^{\prime 2}} \int_{-\psi_{2} / 2}^{-\psi_{1} / 2} \frac{u_{1}(\psi)}{\left(\beta_{4}^{\prime}-1\right)}\left[\left(1+\frac{u_{1}^{\prime 2}}{\left[u_{1}(\psi)\right]^{2}}\left(\beta_{4}^{\prime 2}-1\right)\right)^{\frac{1}{2}}-1\right] d \psi \\
+\frac{B}{\left(1-B \alpha_{2}\right) l u_{1}^{\prime 2}} \int_{\psi_{1} / 2}^{\psi_{2} / 2} \frac{u_{1}(\psi)}{\left(\beta_{4}^{\prime}-1\right)}\left[\left(1+\frac{u_{1}^{\prime 2}}{\left[u_{1}(\psi)\right]^{2}}\left(\beta_{4}^{\prime 2}-1\right)\right)^{\frac{1}{2}}-1\right] d \psi .
\end{gathered}
$$

It is easy to demonstrate that each of the functions $I_{0}, I_{1}$ and $I_{2}$ limit to unity when the upstream flow is uniform; i.e. when $u_{1}(\psi) \rightarrow U$ and $u_{1}^{\prime} \rightarrow U$ for any choice of $\psi_{b}$. In that case, the bypass flow is uniform and (3.12) becomes a simple quadratic function in $\beta_{4}^{\prime}$ that is identical (accounting for differences in notation) to the solution given by Garrett and Cummins (2007) for a disc in uniform flow (see also equation (2.6) given in Draper and Nishino, 2014). Alternatively, for the more general scenario involving a non-uniform velocity profile, $I_{0}$ and $I_{2}$ become functions of the parameter $\beta_{4}^{\prime}$, whilst $I_{1}$ may be evaluated directly. It is therefore possible to use numerical methods to solve both (3.11) and (3.12) to obtain $\beta_{4}^{\prime}$ and $\alpha_{4}$. In this paper we have found this numerical solution for a given velocity profile, geometric blockage ratio and velocity coefficient $\alpha_{2}$ by selecting a range of values for $\beta_{4}^{\prime}$ and computing the corresponding range in values of $\alpha_{4}$ via (3.11). For this range of values the 
functions $I_{0}, I_{1}$ and $I_{2}$ and, in turn, the left-hand side of (3.12) are then evaluated and the relevant root on the interval $[1, \infty)$ is located. A simple bisection method is used to ensure this root is evaluated to within $\sim 10^{-6}$.

Following solution of the velocity coefficients the power extracted by the strip can be evaluated as

$$
P=\int_{-l / 2}^{l / 2} \Delta p(z) u_{2}(z) d z=\int_{-\psi_{1} / 2}^{\psi_{1} / 2} \Delta p(\psi) d \psi
$$

which can be expanded to give

$$
P=\frac{1}{2} \rho\left(1-\alpha_{4}^{2}\right) \int_{-\psi_{1} / 2}^{\psi_{1} / 2}\left[u_{1}(\psi)\right]^{2} d \psi+\frac{1}{2} \rho{u_{1}^{\prime 2}}^{2}\left({\beta_{4}^{\prime}}^{2}-1\right) \int_{-\psi_{1} / 2}^{\psi_{1} / 2} d \psi .
$$

This may also be expressed in terms of a power coefficient such that

$$
C_{P}=\frac{P}{1 / 2 \rho l \overline{U_{*}^{3}}}=\frac{\left(1-\alpha_{4}^{2}\right)}{l \overline{U_{*}^{3}}} \int_{-\psi_{1} / 2}^{\psi_{1} / 2}\left[u_{1}(\psi)\right]^{2} d \psi+\frac{\left(\beta_{4}^{\prime 2}-1\right) u_{1}^{\prime 2}}{l \overline{U_{*}^{3}}} \int_{-\psi_{1} / 2}^{\psi_{1} / 2} d \psi,
$$

where $\overline{U_{*}^{3}}$ is defined in (2.11).

In $\S 4$ we present example solutions to (3.18) for the power removed by a strip for a variety of different velocity profiles. However, before exploring these solutions, we return briefly to the simplification introduced at the start of this section; namely that both $\alpha_{2}$ and $\alpha_{4}$ were assumed to be constant across the area of the strip. As a result of this constraint we can now see from (3.6) and (3.10) that (3.5) can be rewritten as:

$\Delta p(\psi)=\frac{1}{2} \rho\left[u_{1}(\psi)\right]^{2} \times\left(1-\alpha_{4}^{2}+\left[u_{1}(\psi)\right]^{-2} \times u_{1}^{\prime 2}\left(\beta_{4}^{\prime 2}-1\right)\right)$, for $|2 \psi| \leq \psi_{1}$.

Comparing this with (2.8), which defines the pressure difference in terms of the strip resistance, implies that the strip resistance required to ensure a self-similar velocity profile is:

$$
k(\psi)=\frac{1}{\alpha_{2}}\left(1-\alpha_{4}^{2}+\frac{u_{1}^{\prime 2}}{\left[u_{1}(\psi)\right]^{2}}\left(\beta_{4}^{\prime 2}-1\right)\right), \quad \text { for } \quad|2 \psi| \leq \psi_{1} .
$$

This resistance is not constant across the strip, but varies by an amount that is dependent on (i) the shear in the upstream profile intercepting the actuator (defined by $1 /\left[u_{1}(\psi)\right]^{2}$ ) and (ii) the multiplier $u_{1}^{\prime 2}\left(\beta_{4}^{\prime 2}-1\right)$ which, from (3.6), is proportional to the pressure difference across the flow and is significant only for highly blocked flow conditions. The main point to note, therefore, is that when $\alpha_{2}$ and $\alpha_{4}$ are uniform the resistance will be close to uniform when there is minimal shear in the flow intercepting the disc and/or when the geometric blockage is not excessive. In $\S 5$ we present example solutions to demonstrate this.

\section{Solutions for some specific velocity profiles}

\subsection{Symmetric shear flow}

To explore the model presented in $\S 3$, we start by computing the power coefficient for a strip centred at $z=0$ and subjected to a symmetric velocity profile of the form 


$$
u_{1}(z)=U\left(1-2 \frac{|z| B}{l}\right)^{n}
$$

where $n$ is a shape parameter. Exploiting symmetry, it is sufficient to consider this velocity profile in just the lower half of the flow field (i.e. for $z<0$ ). In this half of the flow field (4.1) can be transformed into the $x-\psi$ plane by noting that

$$
\psi(z)+\frac{\psi_{2}}{2}=\int_{-\frac{l}{2 B}}^{z} u_{1}(z) d z=\frac{U l}{2 B(n+1)}\left(1-2 \frac{z B}{l}\right)^{n+1}, \text { for }-\frac{l}{2 B}<z<0,
$$

where $\psi_{2}=U l /(n+1)$ is the total volume flux passing between the lateral boundaries. Using (4.2), equation (4.1) therefore becomes

$$
u_{1}(\psi)=\left(\frac{2 B(n+1)}{l} U^{\frac{1}{n}}\right)^{\frac{n}{n+1}}\left(\frac{\psi_{2}}{2}+\psi\right)^{\frac{n}{n+1}}, \quad \text { for }-\frac{\psi_{2}}{2}<\psi<0 .
$$

We can now substitute this result into equations (3.13), (3.14) and (3.15) to obtain expressions for the functions $I_{0}, I_{1}$ and $I_{2}$ in (3.11) and (3.12). Starting with $I_{0}$ we can write

$$
I_{0}=\frac{2 B \beta_{4}^{\prime}}{\left(1-B \alpha_{2}\right) l} \int_{-\psi_{2} / 2}^{-\psi_{1} / 2}\left(\left[u_{1}(\psi)\right]^{2}+u_{1}^{\prime 2}\left(\beta_{4}^{\prime 2}-1\right)\right)^{-\frac{1}{2}} d \psi,
$$

where the factor of 2 has been introduced due to symmetry and $\psi_{1}$ is the volume flux passing through the strip. This flux can be evaluated by noting that the streamline with $\psi=-\psi_{1} / 2$ passes through the upstream location $z=-\alpha_{2} l / 2$. Consequently, from (4.2) we can write

$$
\frac{\psi_{1}}{2}=\frac{\psi_{2}}{2}-\frac{U l}{2 B(n+1)}\left(1-\alpha_{2} B\right)^{n+1} .
$$

To define $u_{1}^{\prime}$ in (4.4) we are free to choose $\psi_{b}$ at any point in the bypass flow. For example, if we take $\psi_{b}=-\psi_{1} / 2$ (i.e. the edge of the bypass flow) then from (4.5) and (4.3) it follows that $u_{1}^{\prime}=u_{1}\left(-\psi_{1} / 2\right)=U\left(1-\alpha_{2} B\right)^{n}$. Substituting this result and (4.3) into (4.4) now gives

$$
I_{0}=\frac{\beta_{4}^{\prime}}{\left(1-B \alpha_{2}\right)^{n+1}}\left(\frac{2 B}{U l}\right) \int_{-\psi_{2} / 2}^{-\psi_{1} / 2}\left[\left(\frac{\psi_{2}+2 \psi}{\psi_{2}-\psi_{1}}\right)^{\frac{2 n}{n+1}}+\left({\beta_{4}^{\prime}}^{2}-1\right)\right]^{-\frac{1}{2}} d \psi .
$$

Finally, if we introduce a non-dimensional stream function $\tilde{\psi}=\psi(B / U l)$, we can write

$$
I_{0}=\frac{2 \beta_{4}^{\prime}}{\left(1-B \alpha_{2}\right)^{n+1}} \int_{-\widetilde{\psi}_{2} / 2}^{-\widetilde{\psi}_{1} / 2}\left[\left(\frac{\tilde{\psi}_{2}+2 \tilde{\psi}}{\tilde{\psi}_{2}-\tilde{\psi}_{1}}\right)^{\frac{2 n}{n+1}}+\left({\beta_{4}^{\prime}}^{2}-1\right)\right]^{-\frac{1}{2}} d \tilde{\psi},
$$

The integral in this expression does not have an analytical solution for all values of $n$, however it can be evaluated easily numerically for a chosen value of $\beta_{4}^{\prime}$.

Next we can evaluate the two functions $I_{1}$ and $I_{2}$. Firstly, substituting (4.3) into (3.14) and exploiting symmetry leads to

$$
I_{1}=\frac{2}{u_{1}^{\prime 2} \alpha_{2} l} \int_{-\psi_{1} / 2}^{0} u_{1}(\psi) d \psi=\frac{1-\alpha_{2} B}{(2 n+1) \alpha_{2} B}\left[\left(\frac{\tilde{\psi}_{2}}{\tilde{\psi}_{2}-\tilde{\psi}_{1}}\right)^{\frac{2 n+1}{n+1}}-1\right]
$$


(a)

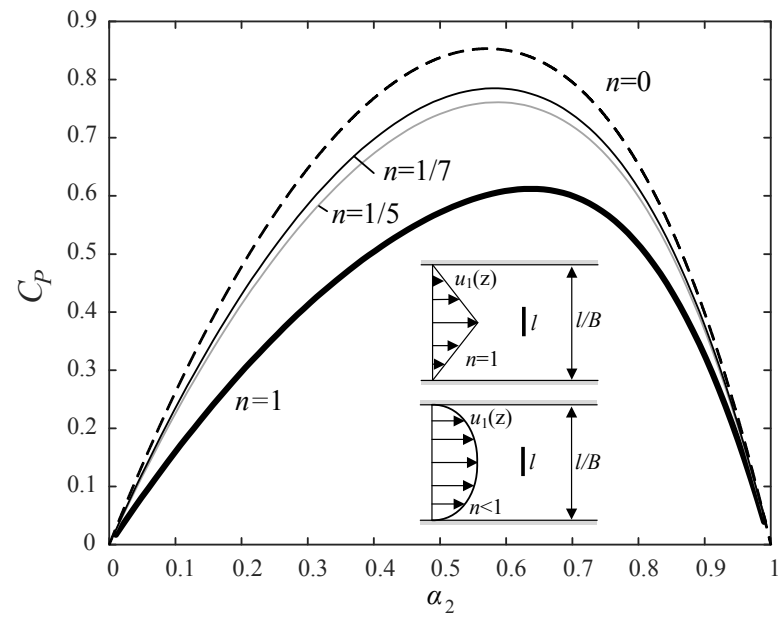

(b)

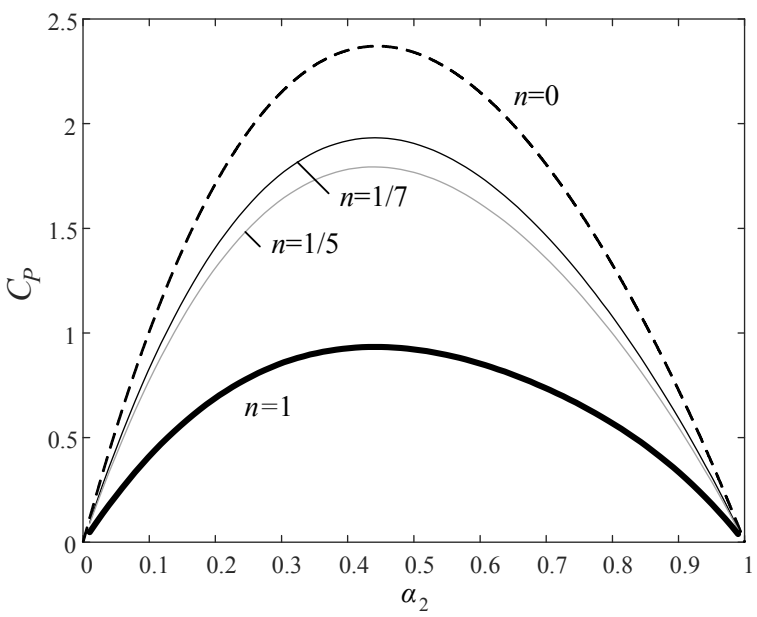

Figure 2: Power coefficient $C_{P}$ for (a) geometric blockage $B=1 / 6$, and (b) geometric blockage $B=1 / 2$. The parameter $n$ defines the shape of the velocity profile given in (4.1).

Secondly, substituting (4.3) into (3.15) leads, after some manipulation, to

$$
\begin{gathered}
I_{2}=\frac{1}{\left(1-\alpha_{2} B\right)^{n+1}} \frac{2}{\beta_{4}^{\prime}-1} \times \\
\int_{-\widetilde{\psi}_{2} / 2}^{-\widetilde{\psi}_{1 / 2}}\left[\left(\left(\frac{\tilde{\psi}_{2}+2 \tilde{\psi}}{\tilde{\psi}_{2}-\tilde{\psi}_{1}}\right)^{\frac{2 n}{n+1}}+\left({\beta_{4}^{\prime}}^{2}-1\right)\right)^{\frac{1}{2}}-\left(\frac{\tilde{\psi}_{2}+2 \tilde{\psi}}{\tilde{\psi}_{2}-\tilde{\psi}_{1}}\right)^{\frac{n}{n+1}}\right] d \tilde{\psi} .
\end{gathered}
$$

Similarly to (4.7), this last function must be evaluated numerically.

For a particular geometric blockage $B$, velocity coefficient $\alpha_{2}$ and shape parameter $n$, it is now straightforward to calculate the unknown coefficients $\beta_{4}^{\prime}$ and $\alpha_{4}$ via (3.11) and (3.12). To do this we adopt the numerical approach outlined in $\S 3$; i.e. we compute $I_{0}, I_{1}$ and $I_{2}$ via (4.7), (4.8) and (4.9) for trial values of $\beta_{4}^{\prime}$ and $\alpha_{4}$. We then use these values to evaluate the left-hand side of (3.12), and repeat the process until obtaining the root.

Having obtained these unknown coefficients we then evaluate the power coefficient. To do this we first note that

$$
\overline{U_{*}^{3}}=\frac{2}{\alpha_{2} l} \int_{-\frac{\psi_{1}}{2}}^{0}\left[u_{1}(\psi)\right]^{2} d \psi=\frac{U^{3}}{\alpha_{2} B} \frac{\left(1-\left(1-\alpha_{2} B\right)^{3 n+1}\right)}{(3 n+1)},
$$

so that (3.18) can be simplified to

$$
C_{P}=\alpha_{2}\left(1-\alpha_{4}^{2}\right)+\alpha_{2}\left({\beta_{4}^{\prime 2}}^{2}-1\right) \frac{(3 n+1)}{(n+1)} \frac{\left(1-\alpha_{2} B\right)^{2 n}\left(1-\left(1-\alpha_{2} B\right)^{n+1}\right)}{1-\left(1-\alpha_{2} B\right)^{3 n+1}} .
$$

Figure 2 presents solutions to (4.11) for two different geometric blockage ratios $(B=1 / 2$ and $1 / 6)$ and for a variety of shape parameters. In this figure the results for $n=0$ correspond to the solution of Garrett and Cummins (2007) for uniform flow. In comparison to these results, it can be seen that the shape of the upstream velocity profile has a significant effect on the power extracted by the strip, with the power coefficient reducing as the flow becomes 
increasingly sheared (i.e. as $n$ increases). For example, for a linear shear profile $(n=1)$, the peak power

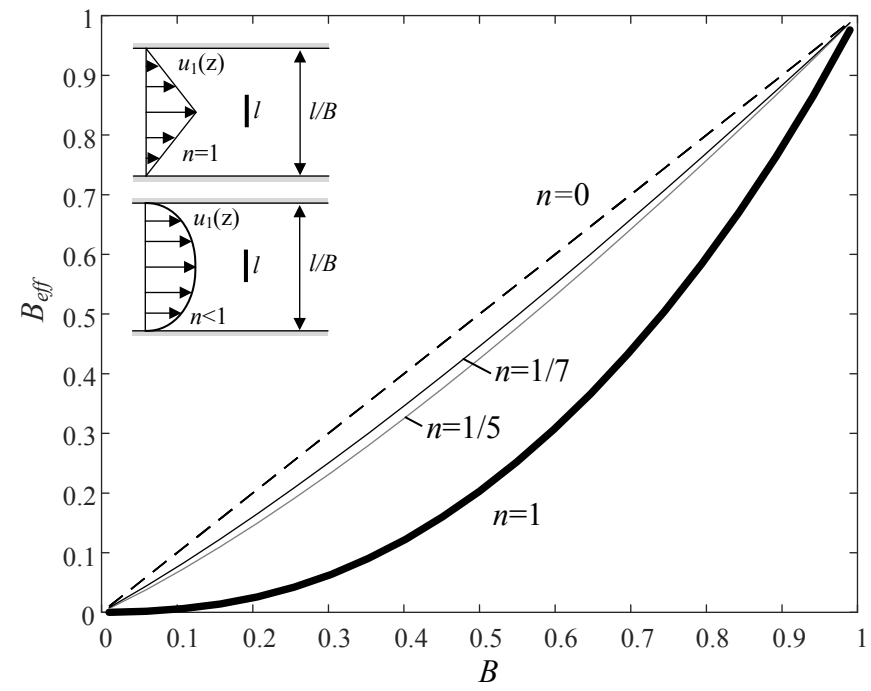

Figure 3: Effective blockage ratio for a strip placed in the centre of a symmetric non-uniform velocity profile. The parameter $n$ defines the shape of the velocity profile given in (4.1).

coefficient is reduced by $61 \%$ and $28 \%$, respectively, compared to the uniform flow solution for $B=1 / 2$ and $1 / 6$.

The reduction in power observed in figure 2 can be understood by realising that the power removed by the strip can increase when there is a large background pressure gradient $\Delta p^{\prime}$, since the product of this gradient and the flow through the channel provides an additional source of power in excess of the upstream kinetic flux (Garrett and Cummins, 2007). However, in a non-uniform flow it can be seen from equation (3.6) that a particular background pressure gradient is achieved when the bypass velocity increases by an amount $\beta_{4}(\psi)=\left[2 \Delta p^{\prime} / \rho\left[u_{1}(\psi)\right]^{2}+1\right]^{1 / 2}$. Hence, when the upstream bypass flow velocity is relatively small, a large fractional increase in the bypass flow velocity is needed to establish a given pressure gradient. Since this increase in velocity requires (from continuity) that a large fraction of the flow must bypass the strip, it is not possible to achieve a large background pressure gradient without a large fraction of the flow bypassing the strip; and this limits the power that can be extracted. Following the same logic, we can also conclude that when the bypass flow velocity is relatively large, only a small fractional increase in velocity is needed to achieve a given background pressure gradient. Consequently a large background pressure gradient can be achieved without a large fraction of the flow bypassing the strip, resulting in increased power extraction (as will be seen in $\S 4.2$ ).

A useful way to quantify the differences in maximum power coefficient observed in figure 2 is to introduce the concept of an effective blockage ratio, which is defined here as the equivalent geometric blockage ratio that would give the same maximum power coefficient in uniform flow. For example, noting that the maximum power coefficient in uniform flow $\left(C_{P, \text { max }}^{U}\right)$ is given by (Garrett and Cummins, 2007)

$$
C_{P, \max }^{U}=\frac{16}{27} \frac{1}{(1-B)^{2}}
$$

it follows that the maximum power coefficient calculated for a non-uniform flow $\left(C_{P, \max }\right)$ can be converted into an effective blockage ratio according to 


$$
B_{e f f}=1-\left(\frac{16}{27 C_{P, \max }}\right)^{1 / 2} .
$$

To explore this concept, the effective blockage ratio is plotted against the geometric blockage ratio for a range of different shape parameters in figure 3 . In this figure it can be seen that the effective blockage is always less than the geometric blockage, except in the limits $B \rightarrow 0$ and $B \rightarrow 1$, where $B_{\text {eff }} \rightarrow B$. The difference between the effective and geometric blockage is most significant for $n=1$, but even for $n=1 / 7$ the effective blockage is more than $10 \%$ lower than the geometric blockage when $B \sim 0.25$ to 0.7 .

\subsection{Asymmetric shear flow}

We now investigate power performance for an asymmetric velocity profile defined by

$$
u_{1}\left(z^{\prime}\right)=U\left(\frac{z^{\prime} B}{l}\right)^{n},
$$

where $z^{\prime}=z+l / 2 B$. Introducing a new stream function coordinate $\psi^{\prime}=\psi+\psi_{2} / 2$, this velocity profile can be transformed into the $x-\psi^{\prime}$ plane by noting that

$$
\psi^{\prime}\left(z^{\prime}\right)=\int_{0}^{z^{\prime}} u_{1}\left(z^{\prime}\right) d z^{\prime}=\frac{U l}{(n+1) B}\left(\frac{z^{\prime} B}{l}\right)^{(n+1)},
$$

so that combining (4.14) and (4.15) gives

$$
u_{1}\left(\psi^{\prime}\right)=\psi^{\prime \prime /(n+1)}\left((n+1) \frac{U^{1 / n} B}{l}\right)^{n /(n+1)} .
$$

In this example we will also allow the centre of the strip to be placed at different lateral locations $z^{\prime}=z_{d}^{\prime}$, where $1 / 2<z_{d}^{\prime} / l<(1-B / 2) / B$ so that the strip remains entirely within the lateral boundaries.

With these input conditions the first step in obtaining a solution is to realise that equations (3.11) and (3.12) are still valid for an asymmetric flow, provided that the integrals in $I_{0}$ and $I_{2}$ are evaluated over the bypass flow and the integral in $I_{1}$ is evaluated over flow passing through the strip. However, having made this realisation, it is immediately evident that (as outlined in $\S 2$ ) the evaluation of these integrals is difficult in practice because the upstream locations of the bypass and core flow are not easy to define in an asymmetric flow. To circumvent this problem we will assume in this example that, to first approximation, the streamlines are not skewed. Consequently, if we define $\psi_{1}^{\prime}$ as the volume flux passing below the strip, and $\psi_{2}^{\prime}-\psi_{1}^{\prime}$ and $\psi_{3}^{\prime}-\psi_{2}^{\prime}$, respectively, as the volume fluxes passing through the strip and above the strip, we can use (4.15) to write

$$
\begin{gathered}
\psi_{1}^{\prime}=\frac{U l}{(n+1) B}\left(\frac{z_{d}^{\prime} B}{l}-\frac{\alpha_{2} B}{2}\right)^{n+1}, \\
\psi_{2}^{\prime}=\frac{U l}{(n+1) B}\left(\frac{z_{d}^{\prime} B}{l}+\frac{\alpha_{2} B}{2}\right)^{n+1} \text { and } \psi_{3}^{\prime}=\frac{U l}{(n+1) B} .
\end{gathered}
$$

Choosing $u_{1}^{\prime}=u_{1}\left(\psi_{1}^{\prime}\right)$ it is now possible to evaluate the integrals in (3.13), (3.14) and (3.15). To do this we again introduce a non-dimensional stream function $\tilde{\psi}_{1}^{\prime}=\psi^{\prime}(B / U l)$ so that, after some manipulation, we can write 


$$
\begin{gathered}
I_{0}=\frac{\beta_{4}^{\prime}}{\left(z_{1}^{\prime} / l\right)^{n}\left(1-\alpha_{2} B\right)}\left\{\int_{0}^{\widetilde{\psi}_{1}^{\prime}}\left(\left(\frac{\tilde{\psi}^{\prime}}{\widetilde{\psi}_{1}^{\prime}}\right)^{\frac{2 n}{n+1}}+\beta_{4}^{\prime 2}-1\right)^{-1 / 2} d \tilde{\psi}^{\prime}+\right. \\
\left.\int_{\widetilde{\psi}_{2}^{\prime}}\left(\left(\frac{\tilde{\psi}^{\prime}}{\widetilde{\psi}_{1}^{\prime}}\right)^{\frac{2 n}{n+1}}+{\beta_{4}^{\prime}}^{2}-1\right)^{-1 / 2} d \tilde{\psi}^{\prime}\right\} . \\
I_{1}=\frac{1}{(2 n+1)} \frac{z_{1}^{\prime}}{\alpha_{2} B l}\left(\left(\frac{z_{2}^{\prime}}{z_{1}^{\prime}}\right)^{2 n+1}-1\right),
\end{gathered}
$$

and

$$
\begin{aligned}
& I_{2}=\frac{1}{\left(z_{1}^{\prime} / l\right)^{n}} \frac{1}{\left(1-B \alpha_{2}\right)} \frac{1}{\beta_{4}^{\prime}-1}\left\{\int_{0}^{\widetilde{\psi}_{1}^{\prime}}\left[\left(\left(\frac{\tilde{\psi}^{\prime}}{\tilde{\psi}_{1}^{\prime}}\right)^{\frac{2 n}{n+1}}+{\beta_{4}^{\prime}}^{2}-1\right)^{1 / 2}-\left(\frac{\tilde{\psi}^{\prime}}{\tilde{\psi}_{1}^{\prime}}\right)^{\frac{n}{n+1}}\right] d \tilde{\psi}^{\prime}\right. \\
& \left.+\int_{\widetilde{\psi}_{2}^{\prime}}^{\widetilde{\psi}_{3}^{\prime}}\left[\left(\left(\frac{\tilde{\psi}^{\prime}}{\tilde{\psi}_{1}^{\prime}}\right)^{\frac{2 n}{n+1}}+{\beta_{4}^{\prime}}^{2}-1\right)^{1 / 2}-\left(\frac{\tilde{\psi}^{\prime}}{\tilde{\psi}_{1}^{\prime}}\right)^{\frac{n}{n+1}}\right] d \tilde{\psi}^{\prime}\right\}
\end{aligned}
$$

where $z_{1}^{\prime} / l=z_{d}^{\prime} B / l-\alpha_{2} B / 2$ and $z_{2}^{\prime} / l=z_{d}^{\prime} B / l+\alpha_{2} B / 2$.

By computing $I_{0}, I_{1}$ and $I_{2}$ using (4.18), (4.19) and (4.20), the unknown parameters $\beta_{4}^{\prime}$ and $\alpha_{4}$ can now be obtained in the same way as that outlined in $\S 3$. After solving for these parameters it is then possible to evaluate the power coefficient via (3.18). To do this we first note that

$$
\overline{U_{*}^{3}}=\frac{1}{(3 n+1)} \frac{U^{3}}{\alpha_{2} B}\left(\left(z_{2}^{\prime} / l\right)^{3 n+1}-\left(z_{1}^{\prime} / l\right)^{3 n+1}\right) .
$$

Hence, (3.18) can be written as

$$
C_{P}=\alpha_{2}\left(1-\alpha_{4}^{2}\right)+\alpha_{2}\left({\beta_{4}^{\prime}}^{2}-1\right) \frac{(3 n+1)}{(n+1)} \frac{\left(z_{1}^{\prime} / l\right)^{2 n}\left(\left(z_{2}^{\prime} / l\right)^{n+1}-\left(z_{1}^{\prime} / l\right)^{n+1}\right)}{\left(\left(z_{2}^{\prime} / l\right)^{3 n+1}-\left(z_{1}^{\prime} / l\right)^{3 n+1}\right)} .
$$

In figure 4 we present the maximum power coefficient based on (4.22) as a function of geometric blockage ratio and strip location for two different shape parameters $(n=1$ and $n=1 / 7)$. In this figure we also plot solutions over the same parameter space for the effective blockage ratio and $C_{p, \max }^{\prime}$, which is the maximum of the power coefficient

$$
C_{P}^{\prime}=\frac{P}{1 / 2 \rho l U^{3}}=\frac{\overline{U_{*}^{3}}}{U^{3}} C_{P}=\frac{1}{(3 n+1)} \frac{1}{\alpha_{2} B}\left(\left(z_{2}^{\prime} / l\right)^{3 n+1}-\left(z_{1}^{\prime} / l\right)^{3 n+1}\right) C_{P} .
$$

Hence, unlike (4.22), the power coefficient $C_{P}^{\prime}$ is not normalised relative to the upstream velocity of the flow passing through the strip and therefore provides a measure of relative power extracted by the strip at different locations in the flow.

Focusing firstly on $C_{P, \max }^{\prime}$ in figure 4 , it is clear that more power is extracted as $z_{d}^{\prime}$ increases; i.e. as the strip is moved to locations where the upstream velocity is largest. In contrast, the normalised power coefficient $C_{P, \max }$ displays the opposite trend, implying a reduction in performance efficiency and a reduction in the effective blockage ratio as the strip is moved to locations where the upstream velocity is fastest. This trend in the normalised power 
coefficient and effective blockage ratio is such that, for both shape parameters, the effective blockage ratio is less than the geometric blockage ratio when the strip is placed higher than a particular location $z_{d, 0}^{\prime}$ (which is indicated by the thick dashed lines in figure 4). Based on the discussion in $\S 4.1$, the trend can be explained by the fact that when the strip is placed at $z_{d}^{\prime}<z_{d, 0}^{\prime}$ the net bypass flow is relatively faster and so comparatively more power can be extracted by the strip than would be predicted using the geometric blockage and assuming a uniform flow. Alternatively, when $z_{d}^{\prime}>z_{d, 0}^{\prime}$, the net bypass flow is relatively slower and power extraction is reduced in the same way as in $\S 4.1$.

For the velocity profiles examined in figure 4 the location $z_{d, 0}^{\prime}$ is generally lower than the solid white lines in figure 4 , which coincide with a location half way between both flow boundaries. This implies that a strip placed in the middle of the flow will have an effective blockage lower than the geometric blockage; the difference being $40 \%$ and $5 \%$ for $n=1$ and $1 / 7$, respectively, when $B=1 / 3$. This has some obvious implications for turbines operating at different locations across highly sheared flows, as discussed further in $\S 6$.

\section{Numerical Modelling}

\subsection{Model background}

To validate the extended actuator disc model we now present numerical simulations of an inviscid shear flow incident on an actuator strip. The numerical code used is outlined in Draper (2011) and solves the shallow water equations (SWEs)

$$
\begin{gathered}
\frac{\partial h}{\partial t}+\nabla \cdot(h \mathbf{u})=0, \\
\frac{\partial u h}{\partial t}+\frac{\partial u^{2} h}{\partial x}+\frac{\partial u v h}{\partial y}=-g h \frac{\partial h}{\partial x}, \\
\frac{\partial v h}{\partial t}+\frac{\partial u v h}{\partial x}+\frac{\partial v^{2} h}{\partial y}=-g h \frac{\partial h}{\partial y},
\end{gathered}
$$

where $\boldsymbol{u}=(u, v)$ defines the velocity components, $g$ is acceleration due to gravity and $h$ is water depth. Although (5.1)-(5.3) represent the SWEs, it is well known that if we set $h(\mathbf{x}, t)=h_{0}+\xi(\mathbf{x}, t)$, in which $\xi(\mathbf{x}, t)$ captures the variation in water depth, and we introduce non- dimensional parameters $h^{\prime}=h / h_{0}, \mathbf{u}^{\prime}=\mathbf{u} / U, \mathbf{x}^{\prime}=\mathbf{x} / l$ and $t^{\prime}=t(U / l)$ for a characteristic length $l$ and velocity $U,(5.2)$ and (5.3) can be rewritten as

$$
\begin{aligned}
& h^{\prime} \frac{\partial h^{\prime}}{\partial x}=-\frac{U^{2}}{g h_{0}}\left(\frac{\partial h^{\prime} u^{\prime}}{\partial t^{\prime}}+\frac{\partial h^{\prime} u^{\prime 2}}{\partial x^{\prime}}+\frac{\partial h^{\prime} u^{\prime} v^{\prime}}{\partial y^{\prime}}\right), \\
& h^{\prime} \frac{\partial h^{\prime}}{\partial y}=-\frac{U^{2}}{g h_{0}}\left(\frac{\partial h^{\prime} v^{\prime}}{\partial t^{\prime}}+\frac{\partial h^{\prime} u^{\prime} v^{\prime}}{\partial x^{\prime}}+\frac{\partial h^{\prime} v^{\prime 2}}{\partial y^{\prime}}\right) .
\end{aligned}
$$

This result indicates that spatial variations in water depth will be negligible when $\mathrm{Fr}=$ $U / \sqrt{g h_{0}} \ll 1$. Hence, in this limit the depth-averaged flow is effectively non-divergent and, since (5.2) and (5.3) can be written as

$$
\frac{\partial \mathbf{u}}{\partial t}+(\mathbf{u} \cdot \nabla) \mathbf{u}=-\nabla g \xi+O\left(\frac{\xi}{h_{0}}\right),
$$

it can be seen that the SWEs become analogous to the incompressible Euler equations (with $\rho g \xi$ representing pressure). Based on this logic we have used the numerical model with $\mathrm{Fr} \leq 5 \times 10^{-3}$ in this work to approximate the incompressible Euler equations. This has 
ensured that spatial variations in $\xi / h_{0}<0.1 \%$ across the numerical domain in all simulations.

To represent the strip we introduce $C u|u|$ to the right-hand side of (5.2) and apply it across a rectangular patch with lateral dimension $l$ and streamwise dimension $s$. The parameter $C$ is set equal to $k h_{0} /(2 s)$, where $k$ is the (uniform) local strip resistance. In 'base case' simulations $s=l / 2$ (although near this value the power is not very sensitive to aspect ratio; see figure 5). The patch is placed $10 \mathrm{l}$ from the upstream boundary of a channel having streamwise length $25 l$ and lateral width that varies according to geometric blockage ratio. The numerical mesh comprises right angled isosceles triangles (with short dimension $l / 2$ ) and fourth order interpolating polynomial basis functions are used (increasing the polynomial order to five, or doubling the mesh, led to differences in power coefficient of less than $2 \%$ ). For all simulations velocity is specified at the upstream boundary, depth is specified at the downstream boundary and symmetry conditions are imposed along the side walls. Initial conditions are $u(x, z)=u_{1}(z)$ and $h(x, z)=h_{0}$. The patch is introduced at $t=0$ and the simulation runs until the power removed by the patch has reached a (near) steady value. This power is calculated by integrating $\rho C|u(x, z)|^{3}$ over the patch area. At the end of the simulation $\alpha_{2}$ is calculated at a given point in the patch based on the streamwise velocity at the point, and the upstream velocity on the same streamline. An average coefficient $\bar{\alpha}_{2}$ was obtained for the line running in the lateral direction through the middle of the patch. In all simulations instability of the wake occurred far downstream $(>5 l)$ of the strip, leading to vortex shedding and a wake qualitatively similar to that for a bluff body with base bleed (Wood, 1964) or a porous obstruction in shallow 
(a)

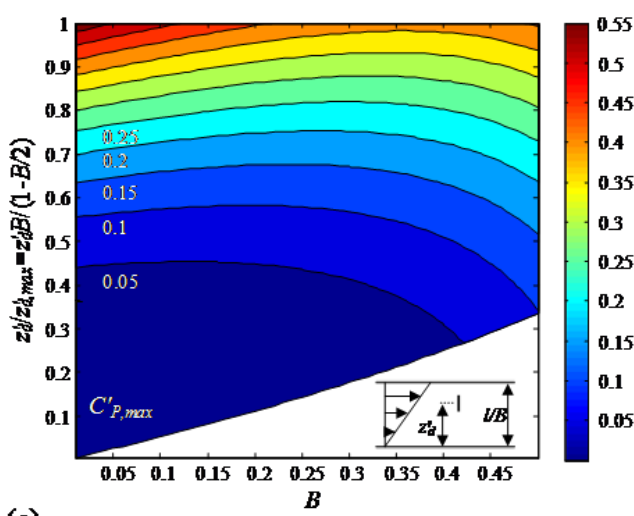

(c)

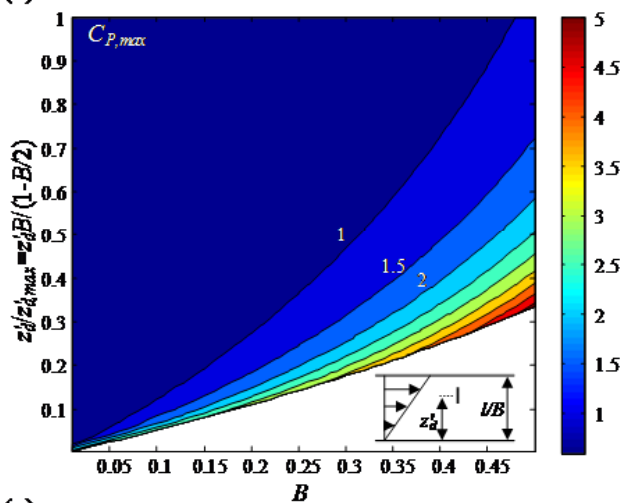

(e)

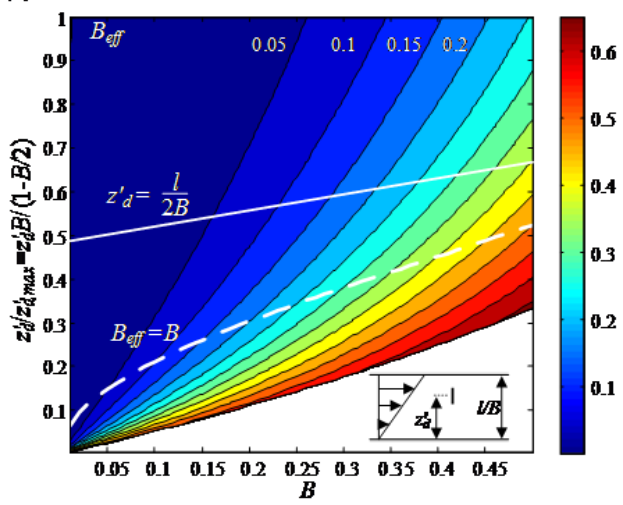

(b)

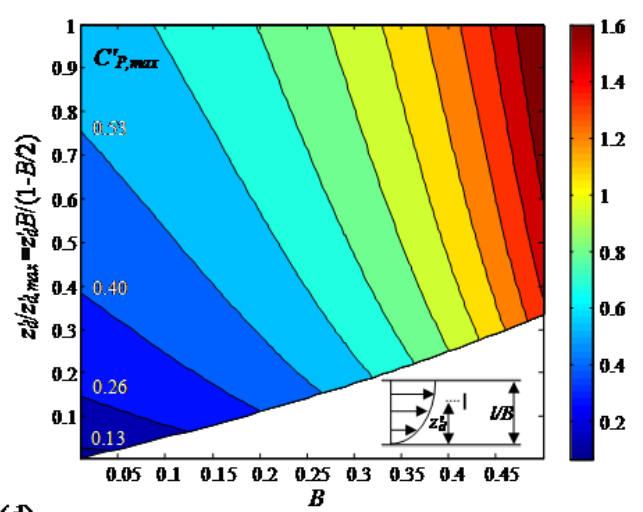

(d)

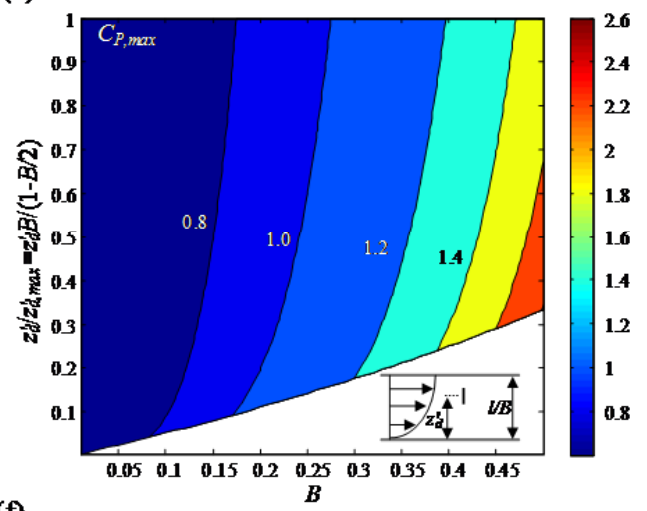

(f)

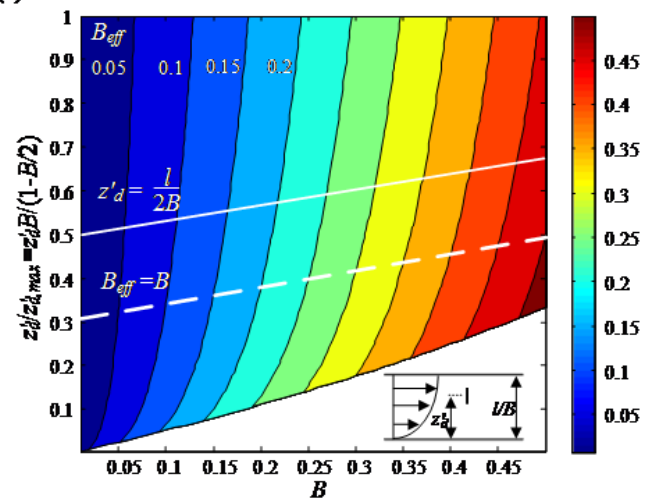

Figure 4: Results for a strip operating in an asymmetric shear flow. (a,b) Maximum power coefficient, normalised by the cube of maximum upstream velocity; (c, d) Maximum power coefficient, normalised by the average of the cube of the upstream velocity passing through the strip; (e, f) Effective blockage ratio. Left hand plots (a, c, e) are for $n=1$. Right hand plots $(\mathrm{b}, \mathrm{d}, \mathrm{f})$ for $n=1 / 7$. 


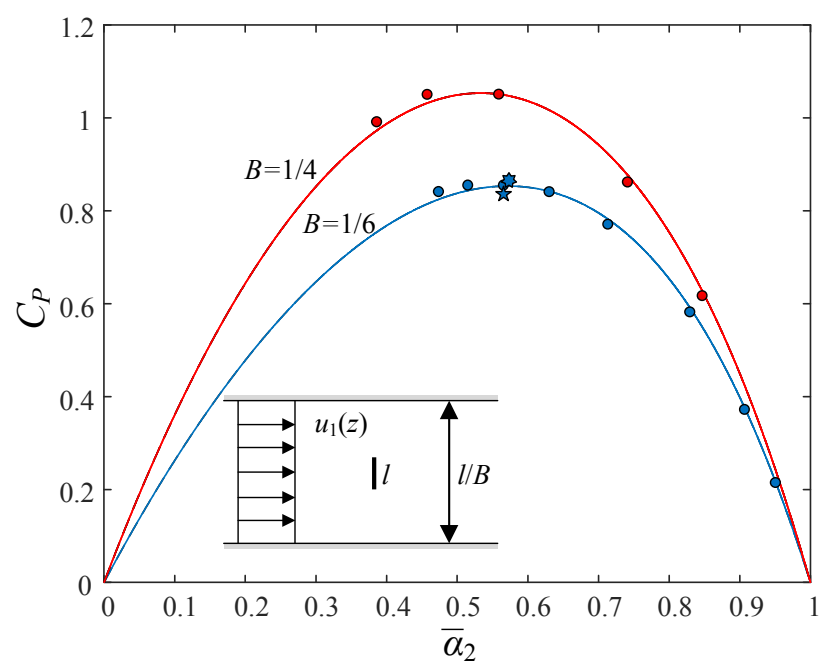

Figure 5: Numerical simulation (markers) and actuator disc solution of Garrett and Cummins (2007) (solid lines). Circles are for standard numerical setup. The marker resembling a 5-point star is for $s / l=1 / 4$; and the marker resembling a 7 point star is for $s / l=1$.

water (Ball et al., 1996). The downstream vortex shedding coincided with small, regular fluctuations in power at the strip (typically $<1 \%$ ), indicating that the downstream wake does affect the flow at the strip, but not significantly.

To investigate performance of the numerical model, figure 5 presents numerical solutions for the power coefficient in uniform flow with $B=1 / 6$ and $B=1 / 4$, and figure 6 a presents an example solution for the flow field. The results in figure 5 are in excellent agreement with the actuator disc solution of Garrett and Cummins (2007).

\subsection{Symmetric shear flow}

We start by comparing the extended model to numerical simulations having upstream velocity equal to (4.1) with $n=1$ (i.e. linear symmetric profile, representative of a more extreme shear flow). For this scenario simulations have been performed for various strip resistances and three geometric blockage ratios $(B=1 / 3,1 / 6$ and 1/10). Computed power coefficients are shown in figure 7 together with predictions from the extended model. It can be seen that the numerical results clearly differ from what might be expected in uniform bounded or unbounded flow and are in very good agreement with the extended model in all cases. The slight under prediction for $B=1 / 3$ is most likely due to the fact that the local resistance is not exactly uniform across the strip in the actuator model at this higher blockage (see figure 10).

Figure $6 \mathrm{~b}$ presents an example solution for a strip with resistance $k=3.5$. This figure illustrates substantial changes to the streamwise velocity profile as the flow encounters the strip. To understand if these changes are captured by the extended actuator model figure 8 presents the streamwise velocity profile for different disc resistances at $4 l$ downstream of the strip, where the streamlines were observed to be almost parallel (indicating pressure has equalised across the flow). Also shown on this figure is the result from the extended model at $x=x_{4}$, which has been calculated according to the parametric equations

$$
u\left(x_{4}, \psi\right)=u_{4}(\psi) \text { and } z\left(x_{4}, \psi\right)=\int_{0}^{\psi} \frac{d \psi}{u_{4}(\psi)}
$$


The agreement between the model result and the numerical simulation in figure 8 is excellent, both in terms of the velocity deficit directly in the wake of the strip and in the bypass flow. The
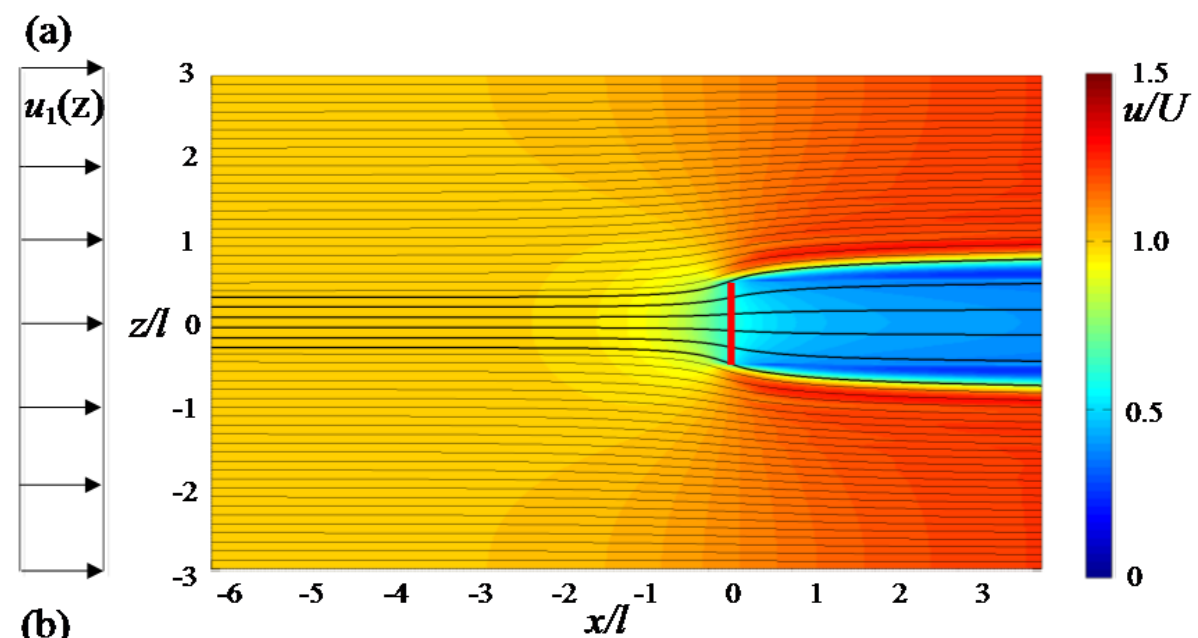

(b)
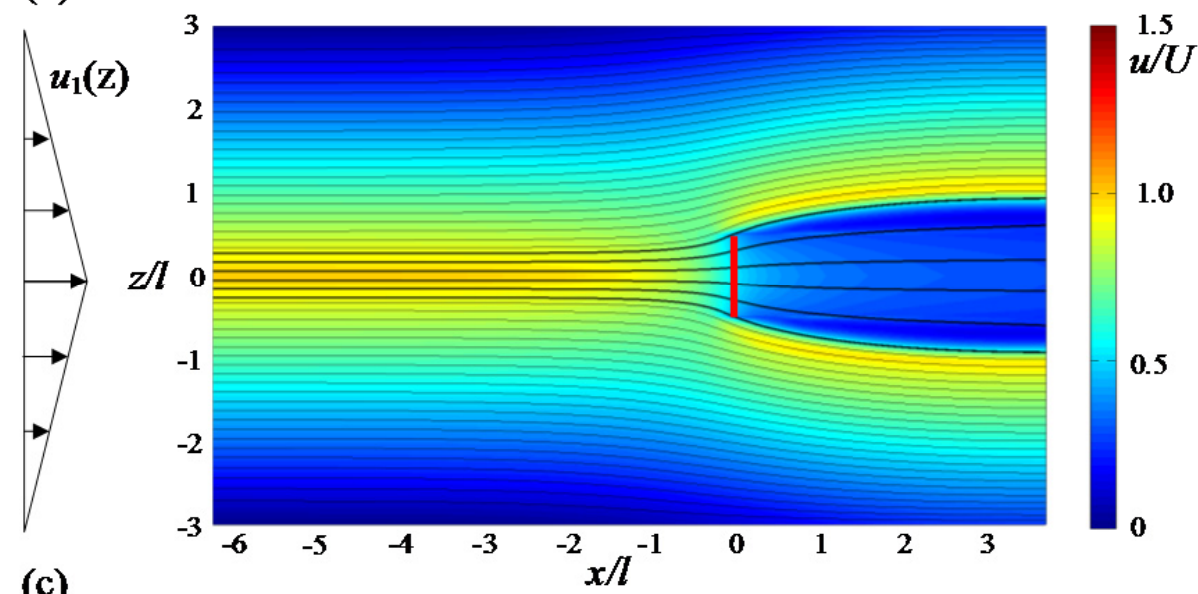

(c)
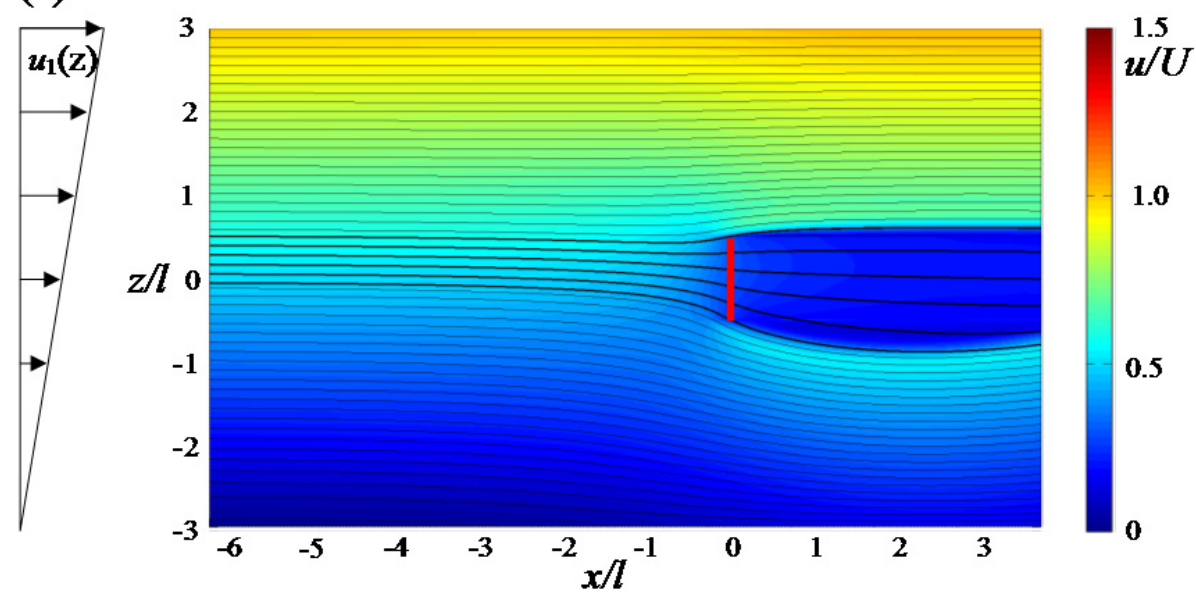

Figure 6: Streamlines and contours of $u(x, z)$ for each type of velocity profile simulated using the numerical model. All strips have $k=3.5$, which correspond to (a) $\bar{\alpha}_{2}=0.63$; (b) $\bar{\alpha}_{2}=0.53$; and (c) $\bar{\alpha}_{2}=0.57$. Vertical (red) line indicates centre of strip.

only region of disagreement between both results is at the transition from the wake to the bypass flow at $z /(l / 2 B) \sim 0.2$ to 0.3 , where the velocity is discontinuous in the actuator model. 
In combination, the comparisons above suggest that the assumption of lateral independence (used in the actuator model) is appropriate. However, to provide more direct justification we interrogate the numerical results further in two ways. First, we investigate if the numerical model shows a uniform local velocity coefficient $\alpha_{2}$ across the strip, since this was predicted

(a)

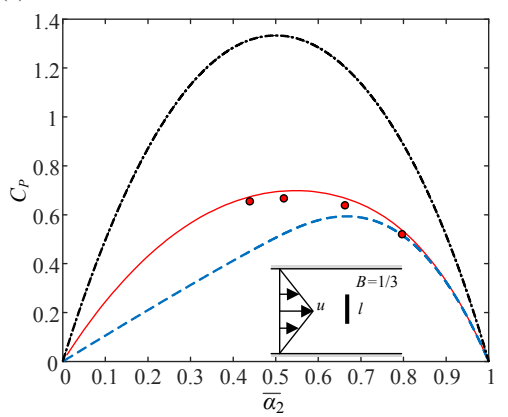

(b)

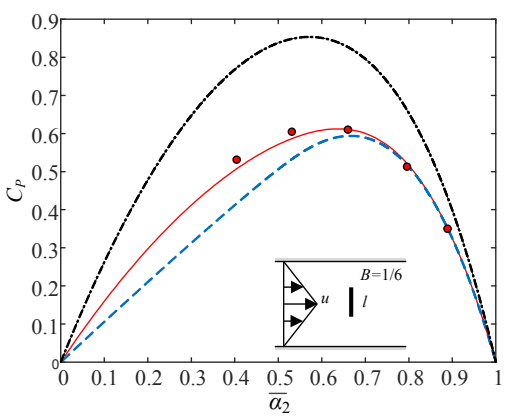

(c)

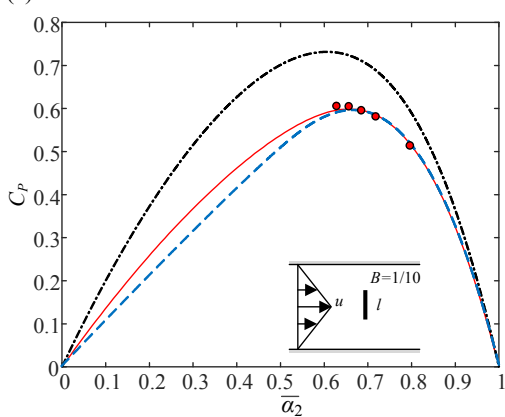

Figure 7: Power coefficient for (a) $B=1 / 3$, and (b) $B=1 / 6$ and (c) $B=1 / 10$. Results from extended actuator strip model (solid lines); numerical simulation results (dots). Actuator model solution also shown for bounded uniform flow (dash dot line) and unbounded uniform flow (dashed line).
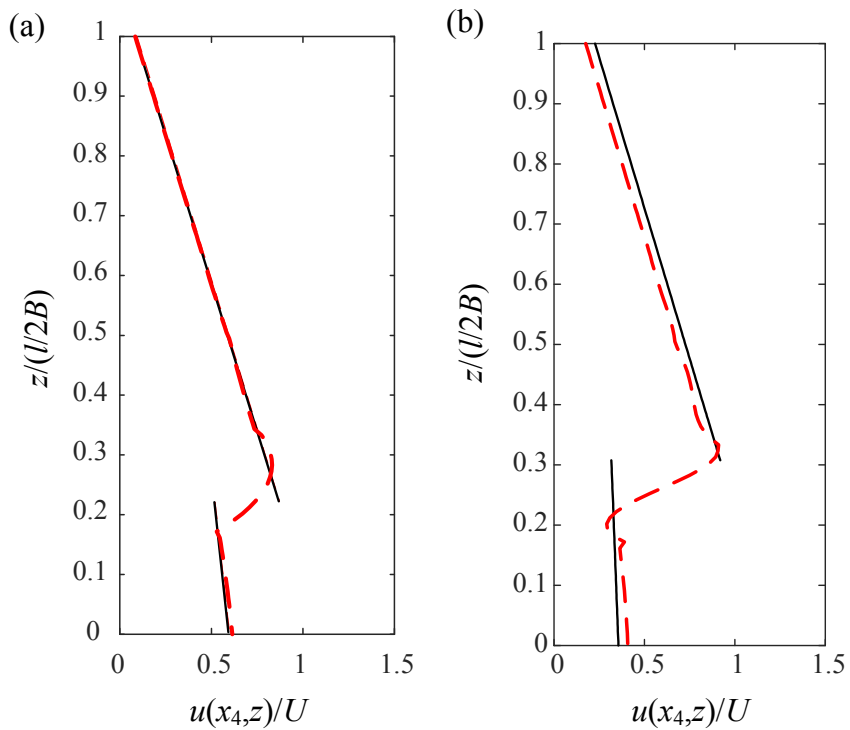

Figure 8: Model profile (solid lines) and simulated profile $4 l$ downstream of strip (dashed lines) for linear symmetric shear flow with $B=1 / 6$. (a) $\bar{\alpha}_{2}=0.80 ; k=1$ (b) $\bar{\alpha}_{2}=0.66 ; k=2$. Only $z>0$ is shown due to symmetry.

in $\S 2$ for a strip with uniform resistance in laterally unbounded flow. For this purpose figure 9 presents $\alpha_{2}$ and the streamwise velocity at the centre of the strip for $B=1 / 10$ and $n=1$ (which is a good approximation to a laterally unbounded flow since it has an effective blockage of $\sim 0.5 \%$ for optimum power extraction; see figure 3 ). It can be seen in figure 9 that $\alpha_{2}$ is indeed close to uniform, with only minor deviations observed at the edge of the strip as $k$ increases.

Second, we can directly compute the forcing $X$, and its density $\delta X$, using the numerical model. To do this we note that for an ideal strip in unbounded flow we can write, using (2.2) and (2.8), that

$$
\left[\alpha_{2}(\psi)\right]^{2} k=\left(1-\left[\alpha_{4}(\psi)\right]^{2}\right)
$$


Combining this with (2.5) therefore gives:

$$
\frac{\delta X}{\delta T}=1-\frac{2}{\alpha_{2}(\psi) k}\left(1-\sqrt{1-k\left[\alpha_{2}(\psi)\right]^{2}}\right)
$$

Hence, noting that by definition $\delta T=\frac{1}{2} \rho k\left[u_{2}(\psi)\right]^{2} \delta l=\frac{1}{2} \rho k u_{2}(\psi) \delta \psi$, it follows that

(a)

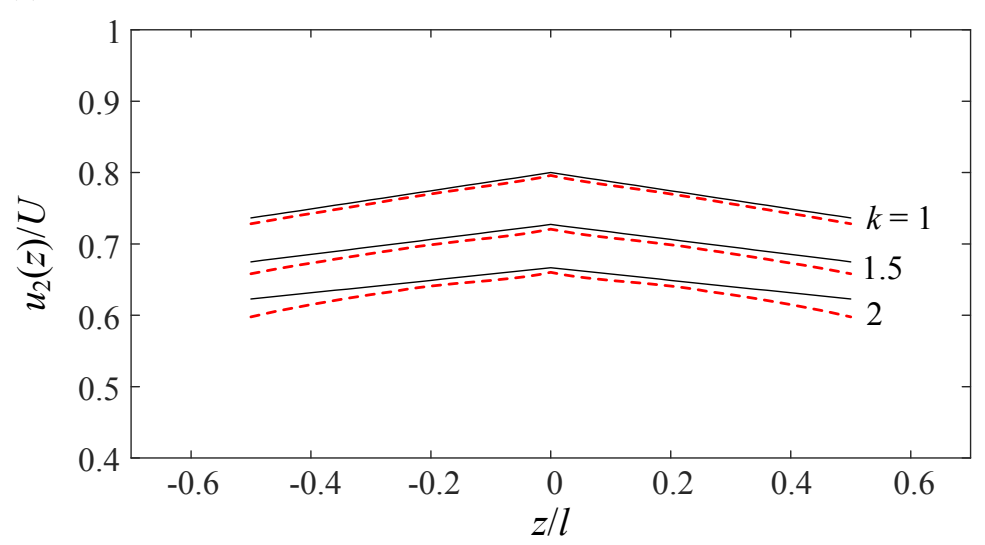

(b)

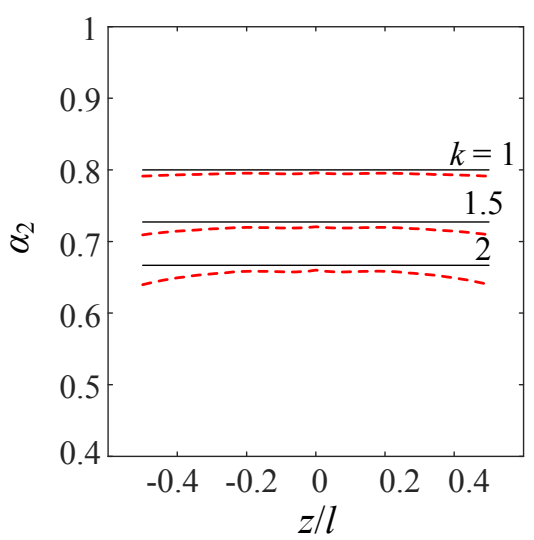

Figure 9: (a) Streamwise velocity at the centre of the strip (normalised by $U$ ) for $B=1 / 10$ and $n=1$. (b) Velocity coefficient $\alpha_{2}$ at the centre of the strip. Extended actuator model (solid lines); Numerical simulation (dashed lines).

$$
X=\int \delta X=\frac{\rho}{2} \int_{-\psi_{1} / 2}^{\psi_{1} / 2} u_{1}(\psi)\left(\alpha_{2}(\psi) k-2\left(1-\sqrt{1-k\left[\alpha_{2}(\psi)\right]^{2}}\right)\right) d \psi
$$

Calculating (5.9) and (5.10) for $n=1$ and $B=1 / 10$ we find that $\delta X / \delta T$ is less than $10 \%$ at all locations across the strip at optimum power extraction, whilst $X / T$ is less than $5 \%$ in the simulations. Thus, lateral independence appears to be appropriate for a strip operating at optimum power coefficient in a flow with a linear (i.e. highly sheared) velocity profile.

Finally, to conclude this section we recall from $\S 3$ that the extended model does not exactly represent a strip with uniform resistance when the blockage is finite and the flow upstream of the strip is non-uniform. To investigate how close to uniform the resistance in the extended model is for the cases presented in this section, figure 10 presents the relevant strip resistances due to (3.20). It can be seen that the resistance is very close to uniform in all cases, and this partly explains the good agreement observed between the actuator model and numerical simulations (especially for $B=1 / 6$ and $1 / 10$ ). 
(a)

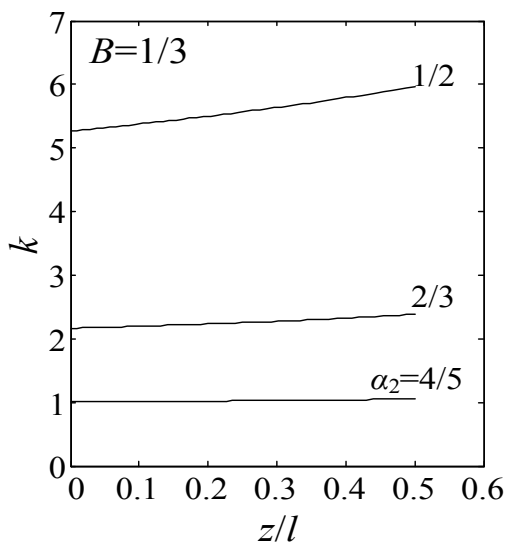

(b)

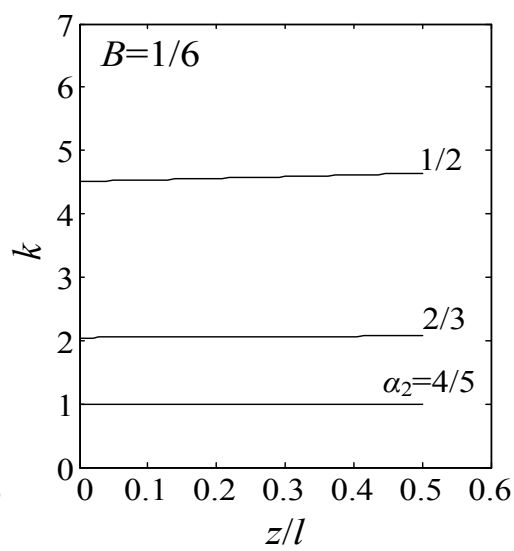

(c)

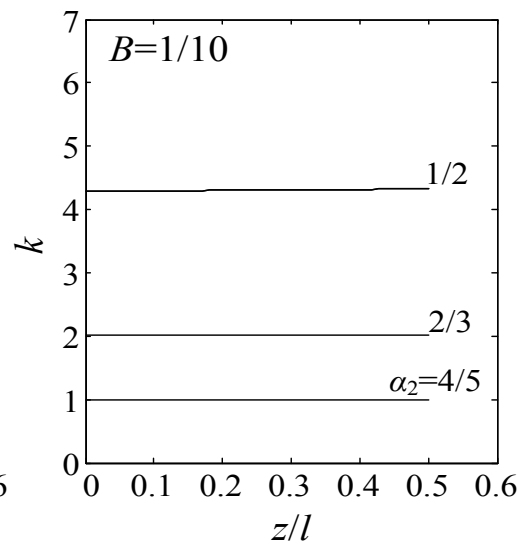

Figure 10: Variation in resistance $k$ across strip in the extended model, computed from (3.20). Only $z>0$ is shown due to symmetry.

\subsection{Asymmetric shear flow}

We now consider an asymmetric velocity profile based on (4.14). Again we choose $n=1$. Numerical simulations are performed for three different strip locations (corresponding to $z_{d}^{\prime} / l=1.5,3$ and 4.5) and geometric blockage $B=1 / 6$.

For these scenarios, simulated power coefficients are presented in figure 11 together with the extended model results. It can be seen in this figure that the extended actuator model under predicts the power compared to the numerical simulations. Figure $6 \mathrm{c}$ and figure 12 show that the reason for this under prediction is that the streamlines are skewed, with the flow through the strip originating from a faster region of the upstream flow than that directly upstream of the strip and assumed in $\S 4.2$. The reason for the deflection of the streamlines is due to the fact that the bypass flow on the side with slower upstream velocity must accelerate by a larger fraction (and therefore, due to continuity, a larger upstream region of the flow must bypass on this side) so as to achieve the same background pressure gradient as that realised by the faster flow bypassing on the other side of the strip. Consequently, the degree of skewing increases when the strip is placed at smaller values of $z_{d}^{\prime}$ (because then the bypass below the strip is relatively slower) and when $k$ is increased (because then the pressure gradient is larger).

A simple way to correct the actuator model predictions to account for skew in the streamlines is to update the location of the strip based on the observed streamline pattern in the numerical simulation (i.e. to increase $z_{d}^{\prime}$ to match the location of the upstream flow). Figure 11 presents a portion of the predicted power curves for each scenario following this correction. It can be seen that the model now does a much better job of matching the numerical simulations, with the agreement within 5-10\% in all cases.

(a)

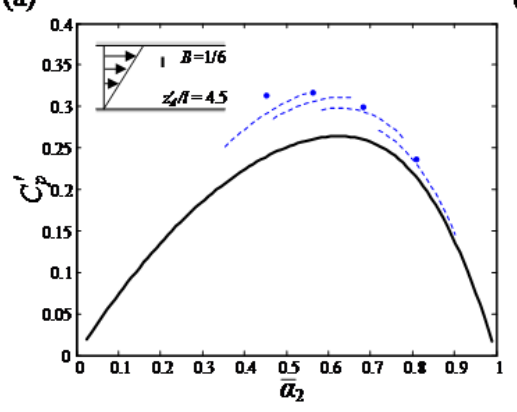

(b)

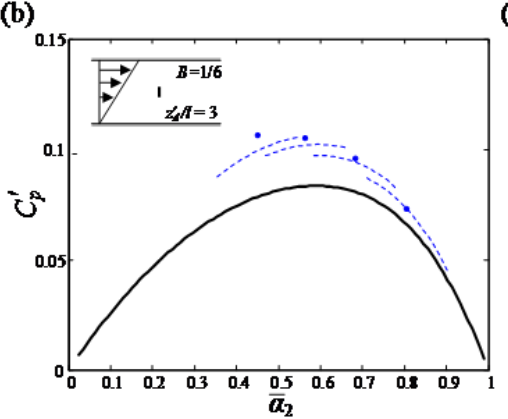

(c)

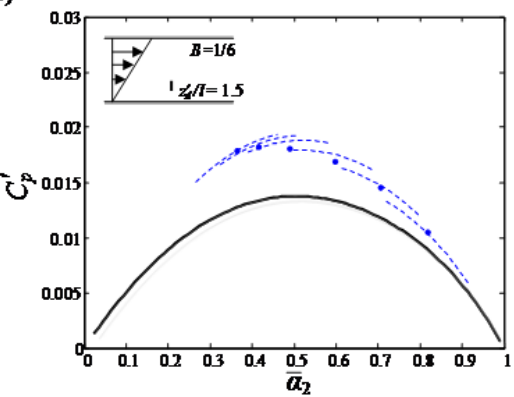


Figure 11: Variation in power coefficient $C_{P}^{\prime}$ for a linear shear flow having $B=1 / 6$. Solid line is prediction based on extended model. Markers show simulation results. Dashed lines are corrected predictions. The strip is located at (a) $z_{d}^{\prime}=4.5 l$; (b) $z_{d}^{\prime}=3 l$; (c) $z_{d}^{\prime}=1.5 l$.

(a)

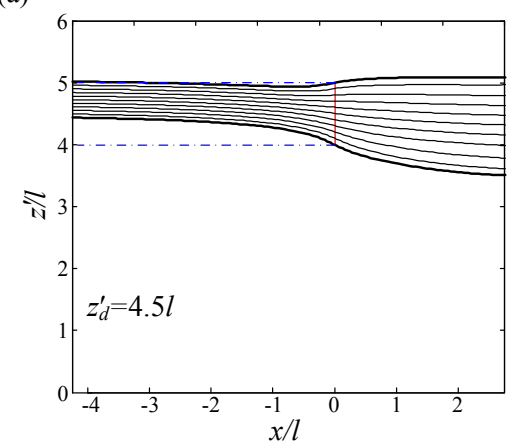

(b)

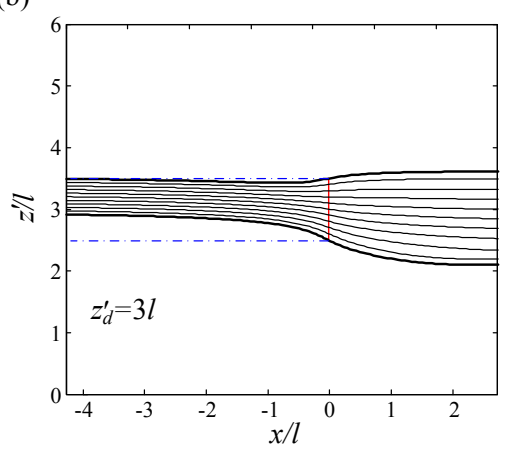

(c)

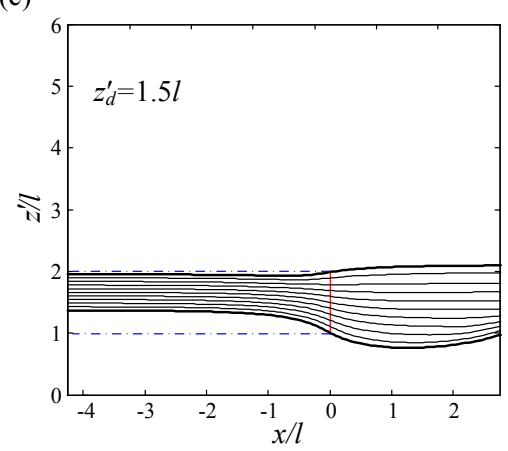

Figure 12: Streamlines passing through the centre of the strip for a linear shear flow, with geometric blockage $B=1 / 6$ and $k=3.5$. The strip is located at (a) $z_{d}^{\prime}=4.5 l\left(\bar{\alpha}_{2}=0.56\right) ;$ (b) $z_{d}^{\prime}=3 l\left(\bar{\alpha}_{2}=0.57\right)$; (c) $z_{d}^{\prime}=1.5 l\left(\bar{\alpha}_{2}=0.59\right)$.

Of course, in practice it would not normally be possible to iterate the theoretical prediction in the way shown in figure 11, because this requires output from a numerical simulation. Hence an assumption of no skew would normally be required in the theoretical model, and this will lead to under-prediction of the power coefficient. For the reasonably extreme case of a linear shear flow this error exceeded $20-30 \%$ for the locations considered in figure 11 . However, in more practical scenarios the error is likely to be lower than this because (i) as noted above, the skewing of the streamlines reduces when the difference in the upstream velocity of the flow bypassing either side of the strip reduces, and so less extreme shear profiles (i.e. $n<1$ ) will lead to less skewing of the streamlines; and (ii) skewing is less prominent when the strip is placed in the faster region of the flow, where power generation is largest.

To complete the comparison between the simulations and the extended model in asymmetric shear flow, figure 13 presents the normalised maximum power coefficient and effective blockage ratio as a function of strip location. In this figure the location $z_{d}^{\prime} / l$ for the numerical results have been adjusted to match the upstream location of the core flow. With this correction it can be seen that there is reasonable agreement with the theoretical model. Additionally the trends shown in these figures are the same as those in figure 4; i.e. as the strip is moved to small values of $z_{d}^{\prime}$, the normalised power and the effective blockage ratio increase by an amount which is dependent on the shape parameter.

\section{Discussion and Conclusions}

In this paper we have extended the classical actuator disc model to investigate the performance of an actuator strip with uniform resistance in laterally unbounded and laterally bounded inviscid shear flow. This model has been compared favourably with numerical simulations approximating the incompressible Euler equations.

For convenience the model has been presented for two-dimensional scenarios. Nevertheless, as noted in $\S 2$, the main results obtained herein for laterally unbounded flow may be applied directly to three-dimensional configurations (as may be most applicable for wind turbines). Additionally, the analysis presented for laterally bounded flows may also be extended in principle to general three-dimensional configurations, provided that the integrations across the individual fluid elements extend across the relevant three-dimensional turbine and bypass flows. In general, however, this will make the analysis more complex than that presented in this paper. For this reason it is therefore relevant to point out that the two-dimensional 
solutions presented in $\S 5$ for laterally bounded flow may be directly useful as approximate solutions for some relevant three dimensional scenarios. Two practical arrangements for which this may be the case are: (i) a long lateral row of closely spaced wind or tidal turbines, in which the shear flow (around each turbine) is confined to the vertical plane, and (ii) a fence of tidal turbines in a wide tidal channel, for which the shear flow (around the entire fence) is confined to the horizontal plane.

With these applications in mind, solution of the extended actuator model has suggested two key results. Firstly, for laterally unbounded flow the model indicates that the power coefficient obtained in different shear flows should collapse to the classical result obtained for uniform flow, provided that the average of the cube of the velocity of the upstream fluid that subsequently passes through the strip is used in the normalisation. This first result is slightly different to that presented in existing literature, and is applicable provided that (i) the local resistance offered by the turbine(s) is uniform and (ii) the upstream location of the flow passing through the turbine(s) can be identified (or estimated). As noted by Fleming et al. (2013), a useful implication of this result is that if the performance of an ideal turbine is known in one particular velocity profile (or location within the velocity profile), the performance of the same turbine in a different shear flow (or location) may be predicted.

(a)

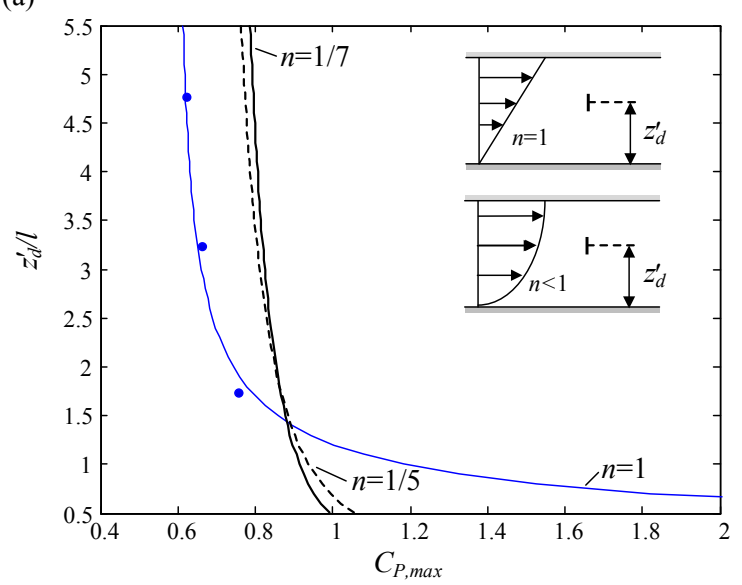

(b)

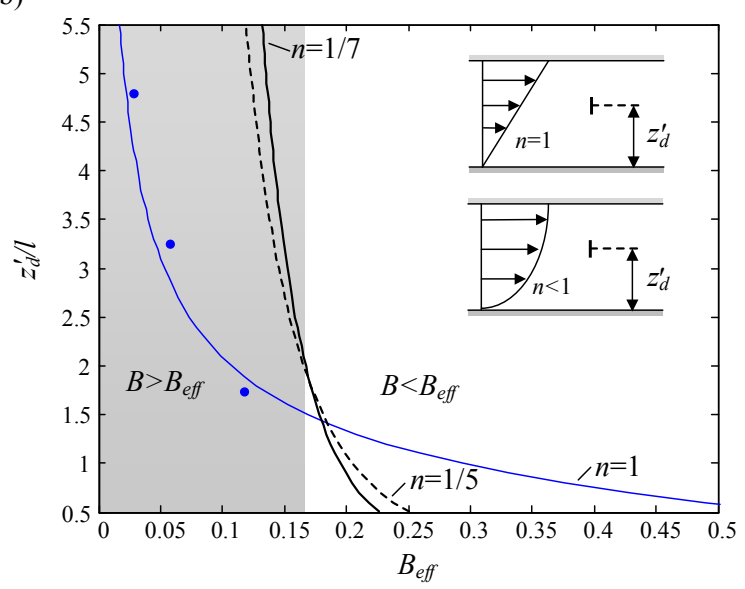

Figure 13: (a) Maximum power coefficient, and (b) effective blockage ratio, as a function of strip location in different asymmetric velocity profiles. Lines represent different shape parameters. Markers indicate numerical simulation results for $n=1$ (plotted with $z_{d}^{\prime} / l$ equal to the upstream location of the core flow observed in the simulations). Shaded region in (b) indicates locations with $B>B_{\text {eff }}$. $B=1 / 6$.

Secondly, for an actuator placed in a laterally bounded shear flow it has been shown that the effective blockage realised by the actuator is dependent on the particular shape of the velocity profile and the relative placement of the actuator. This is because when the upstream velocity of the bypass flow is relatively slow, the background pressure gradient induced due to the blockage is reduced and the power potential is reduced. In the context of the first of the two arrangements mentioned above, which is of particular relevance to tidal turbines, the result also suggests that a row of tidal turbines placed towards the top of the water column in a vertically sheared flow may experience an effective blockage that is significantly less than the geometric blockage. For a $1 / 7^{\text {th }}$ power law velocity profile, for example, the results in $\$ 4.2$ suggest that placing a row of turbines in the top third of the water column can lead to an effective blockage of only $\sim 0.25$ when the geometric blockage is 0.3 (figure 4). Ignoring this reduction in effective blockage (but correcting for the variation in the upstream flow velocity by using $\overline{U_{*}^{3}}$ in the normalisation) would overestimate the power coefficient by $\sim 13 \%$. 
Both of the results above are focused on the power performance of an ideal turbine in an inviscid shear flow. An equally important output for understanding turbine performance in shear flow is the variation in force across the turbine, since variations in force introduce fatigue associated with blade rotation. To this end, the extended model presented herein indicates that the force at any location on the actuator in shear flow is simply (1/ 2) $\rho k \alpha_{2}^{2}\left[u_{1}(\psi)\right]^{2}$, for an ideal turbine with uniform velocity coefficient $\alpha_{2}$ (which, as noted in $\S 3$, implies an approximately uniform resistance $k$ ). This result may be evaluated by solving (3.11) and (3.12), and subsequently (3.20), for a given geometric blockage ratio, upstream velocity profile and velocity coefficient $\alpha_{2}$, and may be used as a first estimate of the mean variation in force across the turbine (or a uniformly porous structure) in shear flow.

Thinking practically, it is also of interest to understand how changes in power performance due to shear effects might compare with effects due to free stream turbulence. At present it is difficult to make this comparison directly for a real turbine rotor; however for ideal turbines represented as actuator discs Nishino and Willden (2012b) have recently shown using threedimensional Reynolds-averaged Navier-Stokes computations that free stream turbulence can enhance near-wake mixing and lead to an increase in power of up to $\sim 10 \%$ across a realistic range of turbulence intensity. Hence it appears that this increase in power may be of a similar order of magnitude to the shear effect investigated in this paper. Interestingly, however, the positive effect of free stream turbulence on power performance may oppose the effects of velocity shear in laterally bounded flows (in which the effective blockage ratio is often lower than the geometric blockage for the majority of turbine placements, as shown in figure 4).

With respect to this comparison between shear effects and free stream turbulence, it is also important to note that the theory presented in this paper ignores the effects of wall friction. When applying the present model to wind or tidal turbine(s) in a vertically sheared flow, for example, bottom friction will act to inhibit bypass flow beneath the turbine. This will lead to less skew in the streamlines passing through the turbine and will act to increase the effective blockage ratio. The importance of these corrections will, however, be negligible if the force due to bed friction is small compared with the force applied by the actuator.

Several extensions to the analysis presented in this paper are possible. For instance, different velocity profiles to those modelled in this paper may be considered, and extending the analysis in this way could build on the work presented in Draper et al. (2014) who modelled a piecewise-constant velocity that was interpreted as a simplified representation of an ocean current. Secondly, it would be worthwhile to extend the analysis in this paper to account for actuator strips with non-uniform resistance in uniform or sheared flows. These strips may be more representative of offshore space-frame structures with variable porosity or realistic turbines modelled using blade element theory. A third extension could take into account wake mixing downstream of the turbine so as to estimate turbine efficiency (or wake loss) in a similar way to Garrett and Cummins (2007) for uniform flow. In a shear flow this particular extension would require some assumption about the downstream velocity profile after mixing, as well as the frictional losses at the lateral boundaries required to establish this profile. Alternatively, if it is assumed that the far downstream velocity profile is the same as that far upstream, and that frictional losses on the lateral boundaries are negligible, control volume arguments may be used directly to provide an upper bound estimate of turbine efficiency.

Finally, we remark that a useful aspect of the extended actuator model presented herein is that it may be used to develop simple corrections on earlier actuator disc models. For instance, the extended model suggests a new refined power coefficient (to directly replace the power coefficient for uniform flow) and the model may be used to define an effective blockage ratio (to directly replace the geometric blockage ratio). The extended model may also be used to 
update existing theoretical models of partial fences and arrays of tidal turbines, such as those developed in Nishino and Willden $(2012 a, 2013)$ and Draper and Nishino (2014), which make use of actuator disc models, but presently assume uniform flow. To do this, the model presented in this paper could be used to determine the relationship between $\alpha_{2}$ and $k$ for an actuator representing a local turbine and/or an array of turbines in these earlier models.

The first author would like to acknowledge the support of the Lloyd's Register Foundation. Lloyd's Register Foundation invests in science, engineering and technology for public benefit, worldwide.

\section{References}

Ball, D. J., Stansby, P. K., \& Allison, N. (1996). Modelling shallow water flow around pile groups. Proceedings of the ICE-Water Maritime and Energy, 118(4), 226-236.

Burton, T., Sharpe, D. Jenkins, N. \& Bossanyi, E. (2001). Wind Energy Handbook. John Wiley \& Sons, Ltd.

Chamorro, L. P., \& Arndt, R. E. (2013). Non-uniform velocity distribution effect on the BetzJoukowsky limit. Wind Energy, 16(2), 279-282.

Draper, S. (2011). Tidal stream energy extraction in coastal basins. DPhil Thesis, University of Oxford.

Draper, S., \& Nishino, T. (2014). Centred and staggered arrangements of tidal turbines. Journal of Fluid Mechanics, 739, 72-93.

Draper, S., Nishino, T. \& Adcock, T.A.A (2014). Turbine Blockage in Non-Uniform Flow. $19^{\text {th }}$ Australasian Fluid Mechanics Conference, Melbourne, Australia.

Fleming, C. F., McIntosh, S. C. \& Willden, R. H. J. (2013). Tidal turbine performance in sheared flow. In Proc. 10th European Wave and Tidal Energy Conference (EWTEC), Aalborg, Denmark.

Garrett, C. \& Cummins, P. (2007). The efficiency of a turbine in a tidal channel. J. Fluid Mech. 588, 243-251.

Goorjian P. M. (1972). An invalid equation in the general momentum theory of the actuator disc. AIAA. 10, 543-44.

Houlsby, G.T., Draper, S. and Oldfield, M. G. L. (2008). Application of linear momentum actuator disc theory to open channel flow. Tech. Rep. OUEL 2296/08, Dept. Engineering Science, University of Oxford.

Myers, L.E. \& Bahaj, A.S. (2012). An experimental investigation simulating flow effects in first generation marine current energy converter arrays. Renewable Energy, 37, 28-36.

Nishino, T. \& Willden, R. H. J. (2012a). The efficiency of an array of tidal turbines partially blocking a wide channel. J. Fluid Mech. 708, 596-606.

Nishino, T. \& Willden, R. H. J. (2012b). Effects of 3-D channel blockage and turbulent wake mixing on the limit of power extraction by tidal turbines. International Journal of Heat and Fluid Flow, 37, 123-135.

Nishino, T. \& Willden, R. H. J. (2013). Two-scale dynamics of flow past a partial cross-stream array of tidal turbines. J. Fluid Mech. 730, 220-244.

Sanderse, B., Pijl, V. D. S., \& Koren, B. (2011). Review of computational fluid dynamics for wind turbine wake aerodynamics. Wind Energy, 14(7), 799-819.

Santo, H., Taylor, P. H., Bai, W., \& Choo, Y. S. (2014). Blockage effects in wave and current: 2D planar simulations of combined regular oscillations and steady flow through porous blocks. Ocean Engineering, 88, 174-186.

Sørensen, J. N. (2011). Aerodynamic aspects of wind energy conversion. Annual Review of Fluid Mechanics, 43, 427-448. 
Taylor, P. H. (1991). Current blockage: reduced forces on offshore space-frame structures. In Offshore Technology Conference. Paper OTC 6519.

van Kuik, G. A. M. (2007). The Lanchester-Betz-Joukowsky limit. Wind Energy, 10(3), 289-291.

Vennell, R. (2010). Tuning turbine in a tidal channel. J. Fluid Mech. 663, 253-267.

Vermeer, L. J., Sørensen, J. N., \& Crespo, A. (2003). Wind turbine wake aerodynamics. Progress in Aerospace Sciences, 39(6), 467-510.

Wagner, R., M. Courtney, M., Gottshall, J., \& Lindelöw-Marsden, P. (2011). Accounting for the speed shear in wind turbine power performance measurement. Wind Energy, 14, 993-1004.

Whelan, J.I., Graham, J.M.R. \& Peiro, J. (2009). A free-surface and blockage correction for tidal turbines. J. Fluid Mech. 624, 281-291.

Wood, C. J. (1964). The effect of base bleed on a periodic wake. Journal of the Royal Aeronautical Society, 68 (1964), pp. 477-482. 